\title{
Prominent local transport in silicon carbide composites containing in-situ synthesized three-dimensional graphene networks
}

Pilar Miranzo ${ }^{a^{*}}$, Laura López-Mirb, Benito Román-Mansoa ${ }^{a}$ Manuel Belmontea ${ }^{\mathrm{a}}$, M. Isabel Osendia and Carmen Ocalb*

a Instituto de Cerámica y Vidrio (ICV-CSIC), Kelsen 5, 28049 Madrid, Spain

b Institut de Ciència de Materials de Barcelona (ICMAB-CSIC), Campus UAB, 08193 Bellaterra, Barcelona,

Spain

*Corresponding Authors: cocal@icmab.es, pmiranzo@icv.csic.es

\begin{abstract}
In-situ grown graphene/SiC composites developed by spark plasma sintering have emerged as a very interesting family of materials with expected high performance for advanced applications. In this work, the local functional properties of graphene/SiC ceramics are elucidated for distinct $\alpha$ - and $\beta$ - SiC polytypes combining scanning probe microscopies. We unambiguously identify all composite constituents and demonstrate the formation of a three-dimensional graphene conductive network inside the composite. The investigated composites exhibit grains with different doping level depending on growth rate during sintering so that conduction paths associated to graphene and matrix networks may compete. The relevance of nanoscale characterization on functional graphene/semiconductor materials is proved as it evidences the type of doping and carrier concentration of the semiconductor and the critical role played by the graphene constituent in the formation of ohmic contacts. Both issues of crucial importance for understanding the macroscale behavior of these materials and determine their applications.
\end{abstract}




\section{Introduction}

For years, silicon carbide $(\mathrm{SiC})$ has raised constant attention because of its multiple applications due to attractive mechanical, thermal and electric properties [1,2]. The interest expands from uses as structural polycrystalline material taking advantage of its thermal, mechanical and tribological properties for friction and wear components, in aerospace applications and for thermal management systems, to functions as a semiconductor single-crystal material in electronics, where its high thermal conductivity, electrical field breakdown strength, and maximum current density make it a promising candidate for high-powered devices at elevated temperatures. As semiconductor element in electronic devices, the capability of changing the $\mathrm{SiC}$ dielectric properties through doping is a very important consideration. In fact, it can be n-type doped by nitrogen or phosphorus and p-type doped by aluminum, boron, gallium or beryllium. Moreover, SiC exists in a large number of polytypes or crystalline forms with diverse characteristics. The most common in commercial uses is the alpha silicon carbide (a-SiC) that refers to hexagonal $(\mathrm{nH}-\mathrm{SiC})$ and rhombohedral polytypes (nR-SiC). The cubic 3C-SiC or beta modification ( $\beta-\mathrm{SiC}$ ) is formed at lower temperatures and has a zinc blende crystal structure (similar to diamond). The electronic characteristics (bandgaps, mobility) of the different polytypes depend on the type and level of doping.

The addition of nanometer sized carbon allotropes (carbon nanotubes and, in particular, graphene) is being nowadays the focus of attention of diverse investigations devoted to improve and modulate the mechanical and electrical performances of these materials for micro-electromechanical devices (MEMs). Akin, the role of epitaxial graphene on $\mathrm{SiC}$ in the characteristics of the solid electrolyte interphase surface [3] and, more recently, the relevance of surface graphitization to activate the electrochemical performances of nanostructured $\mathrm{SiC}$ [4] have demonstrated its potential as anode material in lithium storage batteries. On the one hand, epitaxial graphene (EG) grown on single-crystal SiC substrates by different approaches (CVD, elevated heating...) under ultrahigh vacuum (UHV) conditions has been extensively studied [5-7]. In 
fact, most investigations on thickness controlled graphene creation through elevated heating of SiC have relied on such clean UHV conditions. On the contrary, exploitation of graphene synthesis under non-UHV and, in particular, under the extreme conditions of the spark plasma sintering (SPS) is very scarce. In a previous work [8], we demonstrated that EG formed during densification of SiC powders by SPS method on the surface of the SiC grains due to the low oxygen partial pressure and the high temperature in the SPS chamber aided by the current flowing along the SiC specimens. This interesting phenomena occurred for different $\mathrm{SiC}$ materials including a-(mainly containing $6 \mathrm{H}$ and traces of $4 \mathrm{H}$ and $15 \mathrm{R}$ polytypes) and $\beta-(3 \mathrm{C}$ polytype) SiC, but the kind of $\mathrm{SiC}$ polytype and original powder size had a strong influence over the in-situ formed graphene characteristics (flake size and graphene quality, with a larger amount of disordered stacking in the nano-size powder) and on the electrical conductivity of these graphene/SiC composites. For instance, the amount of defects in the formed graphene depended on the original SiC grain size, whereas the electrical conductivity of the composites depended on both the polytype and the SiC grain size, varying from $8.3 \times 10^{-5} \mathrm{~S} \cdot \mathrm{m}^{-1}$ for the $\mathrm{a}-\mathrm{SiC}$ material to $1.02 \times 10^{2} \mathrm{~S} \cdot \mathrm{m}^{-1}$ for the nanosized $\beta$-SiC (see Table 1 where the properties and characteristics of these composites are collected). More recently, results on the contactmechanical properties at pre-creep temperatures $\left(850^{\circ} \mathrm{C}\right)$ for these composites have demonstrated quantitative differences associated to the microstructure [9]. In order to further explore the capabilities of the SPS method to design and fabricate SiC materials with tunable conductivity for high performance electromechanical micro-devices, we explore here the local properties of such graphene based nanocomposites evidencing the key roles of polymorphism and initial size of the SiC ceramic powders, and their doping on the microscale conducting behavior. This new understanding is of paramount importance to straightly link the nanoscale behavior to the macroscale performance of these materials in different applications either for friction purposes or for electronic devices and batteries.

The intrinsically nanometric dimensions of the graphene related materials makes the use of nano-scale scanning probes particularly worthy for their characterization. A combination of Scanning Force 
Microscopies (SFM) has been employed for differentiating primary and secondary phases in SiC ceramics, distinguishing the graphene component and determining its spatial distribution, as well as elucidating the electrical and frictional responses of each composite constituent. The capabilities of this combined strategy have been successfully employed for the study of laterally heterogeneous surfaces exhibiting coexistence of surface regions with clearly differentiated electrical properties $[10,11]$. SFM in its lateral force mode (also known as Friction Force Microscopy, FFM) has been used to identify the diverse constituents via their different mechanical responses. Other SFM variants using conductive probes, namely conducting SFM (CSFM) and Kelvin probe force microscopy (KPFM), have been employed to obtain the local electric current and surface potential (or contact potential difference, CPD) without changing surface location and, therefore, allowing unambiguous correlation of all these magnitudes for each constituent. The electrical response of the in-situ graphene flakes in these composites indicates that they are connected and form a conducting three-dimensional (3D) network, whereas the SiC matrix shows a semiconductor character with n-type or p-type doping depending on the particular composite.

\section{Materials and methods}

$\mathrm{SiC}$ materials were prepared from three different starting powders: $a-S i C$ (S-2022, CERAC, $d_{50}=0.78 \mu \mathrm{m}$, polytype $6 \mathrm{H}$ ), $\beta-\mathrm{SiC}$ (BF-17A, HC-Starck, $d_{50}=0.5 \mu \mathrm{m}$, polytype $3 \mathrm{C}$ ), and nano- $\beta-S i C$ (NanoArmor, NanoArmor, $d_{50}=45-55$ nm, polytype $3 C$ ), using 5 wt.\% of $\mathrm{Y}_{2} \mathrm{O}_{3}$ (Grade C, H.C. Starck GmbH \& Co., Germany) and 2 wt.\% of $\mathrm{Al}_{2} \mathrm{O}_{3}$ (SM8, Baikowski Chimie, France) as sintering additives. The $\mathrm{SiC}$ and additive powders were mixed by attrition milling in ethanol for $2 \mathrm{~h}$ using $\mathrm{Si}_{3} \mathrm{~N}_{4}$ grinding media. Solvent was evaporated from the suspensions in a rotary-evaporator at $90^{\circ} \mathrm{C}$ and the resultant mixture was oven dried at $120^{\circ} \mathrm{C}$ and then sieved through a $63 \mu \mathrm{m}$ mesh. The mixtures were spark plasma sintered (SPS-510CE, SPS Syntex Inc., Japan) into discs of $20 \mathrm{~mm}$ in diameter and $3 \mathrm{~mm}$ thickness using a heating rate of 133 
${ }^{\circ} \mathrm{C} \cdot \mathrm{min}^{-1}$, an uniaxial pressure of $50 \mathrm{MPa}$ and a holding time of $5 \mathrm{~min}$, under $4 \mathrm{~Pa}$ of vacuum atmosphere, at temperatures ranging from 1800 and $1850^{\circ} \mathrm{C}$. The sintered specimens were cut into slabs and polished using silicon carbide paper and successive diamond compounds as the standard procedure to get mirror finished surfaces.

Micro-Raman maps were recorded on cross sections of the different specimens by confocal Raman-atomic force microscopy (AFM, model Alpha300 WITec GmbH, Germany), using the $532 \mathrm{~nm}$ laser wavelength excitation, and an acquisition up to $3000 \mathrm{~cm}^{-1}$. The microstructure of the specimens was observed by field emission scanning electron microscopy (FESEM, S-4700, Hitachi, Japan) of polished and plasma etched surfaces.

Scanning force microscopy (SFM) measurements were performed under low humidity conditions $(<5 \% \mathrm{RH}$, obtained by a continuous $\mathrm{N}_{2}$ gas flux) using a commercial head and software from Nanotec [12]. Borondoped diamond coated Si probes were used for all SFM measurements presented here. These tips exhibit a good electrical conductivity and resistance to wear, making them appropriate for hard samples analysis. On the other hand, their contact resistance (about ten $\mathrm{k} \Omega$ on metallic surfaces) is adequate for experiments on resistive samples. An intermediate cantilever spring constant $\left(\mathrm{k}=3.0 \mathrm{~N} \mathrm{~m}^{-1}\right)$ was chosen as a compromise to have the same probe for contact SFM operation (FFM and CSFM) and dynamic imaging during KPFM. In this work, FFM [13] has been used to assess the chemical differentiation between composite constituents during contact mode measurements. CSFM [14] was used to obtain direct tip-surface conducting response whereas KPFM $[15,16]$ in the lift mode was used to accurately determine local variations in surface contact potential difference $(\Delta S P)$. For each sample, distinct nanoscale characterizations were made on exactly the same locations to correlate local properties for the different constituents. Tip-sample conditions were systematically checked by force versus distances curves. The same tip was used in all the experiments of 
at least one series. Experimental details of these measuring modes and the particular set-up employed are given in the Supplementary Data and can be found elsewhere [17-20].

\section{Results and discussion}

The characterization of the investigated composites by XRD [8] revealed that no phase transformation occurs during sintering and that the secondary phase crystallizes as $\mathrm{Y}_{3} \mathrm{Al}_{5} \mathrm{O}_{12}(\mathrm{YAG})$ or $\mathrm{YAIO}_{3}(\mathrm{YAP}$ ) in both graphene/a-SiC (G/a-SiC) and graphene/ $\beta-S i C(G / \beta-S i C)$ composites. Conversely, it remains amorphous for the graphene/nano- $\beta-\mathrm{SiC}(\mathrm{G} / \mathrm{n} \beta-\mathrm{SiC})$ material owing to the higher oxygen content of the original powders (i.e., 4.1 wt.\% oxygen in $n \beta-S i C$ vs 1.2-1.7 wt.\% in a-SiC and $\beta-S i C$, respectively) [8], therefore shifting the grain boundary phase composition to the glass-forming region of the $\mathrm{Al}_{2} \mathrm{O}_{3}-\mathrm{Y}_{2} \mathrm{O}_{3}-\mathrm{SiO}_{2}$ phase equilibrium diagram [21]. In addition, the higher content of liquid phase developed at the sintering temperature for $\mathrm{G} / \mathrm{n} \beta$ SiC compared to the other two composites (i.e., $14.2 \mathrm{wt.} \%$ vs $~ 10.0 \mathrm{wt} . \%$ ) [8], importantly enhances grain growth kinetics so that the final SiC grain size increases from the starting mean value $\sim 0.050( \pm 0.005) \mu \mathrm{m}$ up to $\sim 0.400 \mu \mathrm{m}$ upon sintering. For this material, a high level of $p$-doping is expected since grain growth during liquid phase sintering of the ceramics takes place by a solution-precipitation process in which atoms of the additives, aluminum and yttrium in this case, enter the SiC grains forming a solid solution rim that controls the electrical conductivity response of these polycrystalline materials. Conversely, SiC grain size in G/a-SiC and G/B-SiC $(1.11 \mu \mathrm{m}$ and $0.54 \mu \mathrm{m}$, respectively) remains close to the starting values $(0.80 \mu \mathrm{m}$ and $0.50 \mu \mathrm{m}$, respectively) and, therefore, lower doping levels are expected for them. Representative FESEM images of the microstructure of the three materials are shown in Fig. 1, where the grains size differences among them are clearly perceived. 

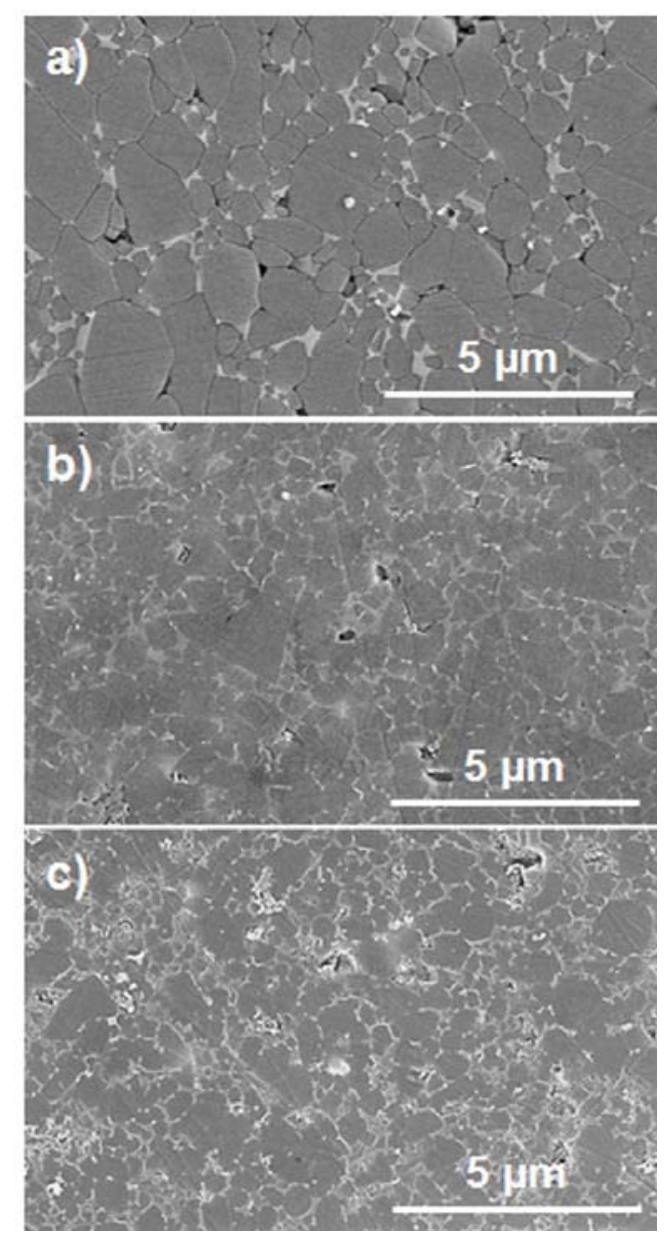

Fig. 1. Representative FESEM micrographs of the polished and plasma etched surface of (a) G/a-SiC, (b) G/ß-SiC and (c) G/nß-SiC composites.

The sub-micrometric morphology of the mirror like polished G/a-SiC composite surface is shown in Fig.2a and consists of extremely flat areas where the fine straight polishing lines conceal the grain microstructure. Conversely to this relatively featureless topography, lateral force images (Figs. $2 \mathrm{c}$ and $2 \mathrm{~d}$ ) reveal dramatic contrast indicating the presence of three species with different mechanical properties. According to the interpretation of FFM imaging (see Supplementary Data) darker/brighter contrast in the forward image (Fig. 2c) indicates lower/higher friction. Therefore, regions with no defined shape and undistinguishable in topography (e.g. black arrows) have a considerably lower friction than the surrounding surface (a-SiC phase) whereas material protruding from the surface plane (e.g. white arrows) presents higher friction (see 
also Figs. S1 and S2 in the Supplementary Data). As it has been already reported for similar [8] and other as-polished graphene-based ceramic composites [22], namely graphene/Si $\mathrm{N}_{4}$, the protruding material corresponds to the graphene flakes at the $\mathrm{SiC}$ grain boundaries that constituting a $3 \mathrm{D}$ network within the bulk composite result exposed to the surface after cutting and polishing. Analysis of friction for diverse few layer graphene (FLG) outcropping at inter-grain sites as a function of thickness and tip scanning direction (see Figs. S3 and S4 in the Supplementary Data) reveals that weakly bounded graphene exhibits a much larger friction than strongly attached layers and the thicker the flake the lower the friction. These observations agree well with results on the structural and frictional properties of isolated FLG, either epitaxialy grown on $\mathrm{SiC}$ single-crystals or transferred (after mechanical cleavage of graphite) to $\mathrm{Si}$ or $\mathrm{SiO}_{2}$ substrates [23-27] indicating that the frictional force decreases with increasing number of graphene layers.

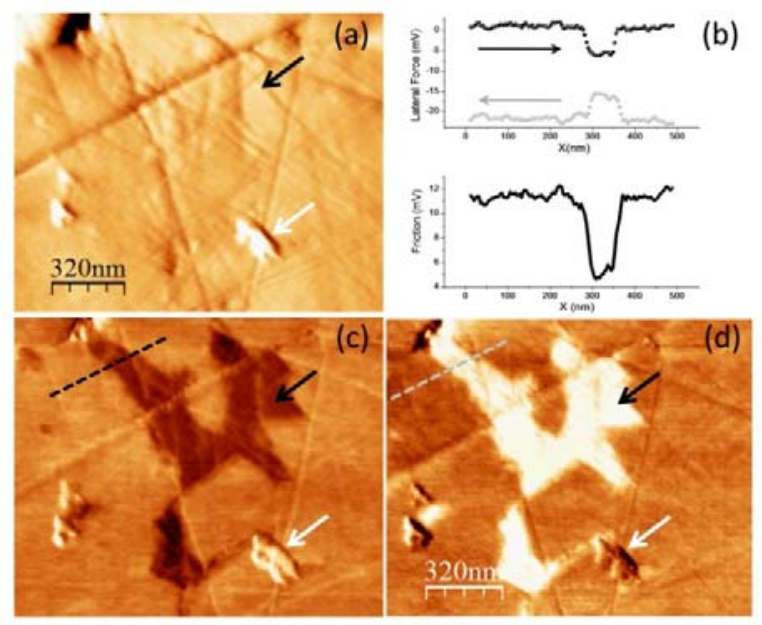

Fig. 2. Topography (a) and lateral force and friction data (b,c,d) for G/a-SiC composite. Black and grey lateral force profiles in the top panel of (b) are taken along the corresponding dashed segments in the forward (c) and backward (d) lateral force images and are used to calculate the friction difference shown in the bottom panel of (b) between majority SiC and secondary phase. All images are $1650 \mathrm{~nm} \times 1340 \mathrm{~nm}$ and the total vertical scale in (a) is $10 \mathrm{~nm}$. The secondary phase has a $52 \%$ lower friction than the a-SiC primary phase.

The material with the lowest friction observed in Fig. 2 (dark and bright patches signaled by black arrows in Figs. $2 c$ and $2 d$, respectively) has a calculated friction (Fig. $2 b$ ) of about $50 \%$ lower than the majority a-SiC phase. Though, in general, direct relationship between friction and crystallinity remains unclear, we ascribe 
these low friction regions to the crystalline secondary phase (YAG) in $\mathrm{G} / \mathrm{a}-\mathrm{SiC}$ because they constitute an estimated $\sim 5 \%$ areal fraction of the overall surface (see Fig. S1 in the Supplementary Data) in excellent agreement with the added volume fraction of secondary phase $(\sim 5$ vol. \%) in this composite [8].

As a novelty and for a comprehensive description of the local properties, KPFM and CSFM have been combined here with FFM in the very same sample region of the composites. During the past years, KPFM has been used to provide thickness dependent charge screening and local doping in exfoliated graphene supported on $\mathrm{SiC}$ and $\mathrm{SiO}_{2}$ substrates [28-32] and in graphene films grown on $\mathrm{SiC}(0001)$ by thermal decomposition synthesis [23, 33-36]. CSFM has demonstrated powerful capability for measuring local current in epitaxial and exfoliated graphene systems [37-41] and in different types of composites containing graphitic networks, e.g. G/polymer composites [42], and $\mathrm{G} / \mathrm{SiC}$ [8], $\mathrm{G} / \mathrm{Si}_{3} \mathrm{~N}_{4}$ [22], and $\mathrm{CNTs} / \mathrm{Si}_{3} \mathrm{~N}_{4}$ [43] ceramic composites.

As it can be observed in Fig. 3, there is a strong correlation between topography (Fig. 3b), lateral force (Fig. 3c), current (Fig. 3d), and surface potential (Fig. 3g) images of the same G/a-SiC specimen. Fig. 3a shows the topographic relief of a graphene sheet $(\sim 4 \mathrm{~nm})$ taken along the segment drawn in the topographic image of Fig. 3b. This particular flake and some regions ascribed to the secondary phase can be easily identified in the lateral force images of the selected area shown in Fig. 3c. For the applied voltage $\left(V_{\text {tip }}=+1\right.$ V) current readout is obtained only over graphene flakes (Fig. 3d), which show a linear symmetric I-V response as corresponds to ohmic elements (Fig. 3e). Because of our CSFM set-up, in which the current is measured using a macroscopic electrode placed millimeters apart from the tip-sample contact (see Supplementary Data), the fact of detecting current at graphene sheets embedded between the matrix grains indicates that these flakes are electrically connected and form part of a conducting network within the composite. The heterogeneities in the current map arise from the quality of each particular flake as demonstrated by additional measurements (Figs. S5 and S6 in the Supplementary Data). It is noticeable 
that, though no current is measured on the rest of the composite surface at the applied voltage $(+1 \mathrm{~V}), \mathrm{I}-\mathrm{V}$ curves obtained for a voltage range $\pm 10 \mathrm{~V}$ at the primary SiC phase show a clear n-type rectifying behavior (Fig. 3f).

Comparison between all images permits unambiguously identifying the KPFM signals in Fig. $3 g$ as corresponding to the secondary phase (I), large areas of the majority a-SiC phase (II) and graphene flakes (III). In addition, small regions with an intermediate color shade (IV) are not correlated to topographic relief or friction contrast. These observations have been verified over all analyzed surface locations (Fig. S7 in the Supplementary Data). The work function of the metallic B-doped diamond coated tip ( $\phi_{\text {tip }} \sim 4.75 \mathrm{eV}$ ) is the reference for KPFM values $(\triangle S P=0)$. Provided the surface potential measured by KPFM is directly related to the carrier concentration, influenced by the type and level of doping we argue that regions IV correspond to $\alpha$-SiC grains with distinct doping level. In Fig. $3 \mathrm{~h}, \Delta \mathrm{SP}$ over graphene is nearly zero, pointing to a work function close to that of bulk graphite $(\sim 4.8 \mathrm{eV})[44]$. On the other hand, $\Delta \mathrm{SP}$ is of $\sim 475 \mathrm{mV}$ and $\sim 260 \mathrm{mV}$ for the secondary and majority $\alpha$-SiC phases, respectively, while the doped grains have about $100 \mathrm{mV}$ lower $\Delta \mathrm{SP}$ than the majority phase and, in consequence, are expected to have a higher conductivity. This hypothesis is demonstrated next. 
(a)
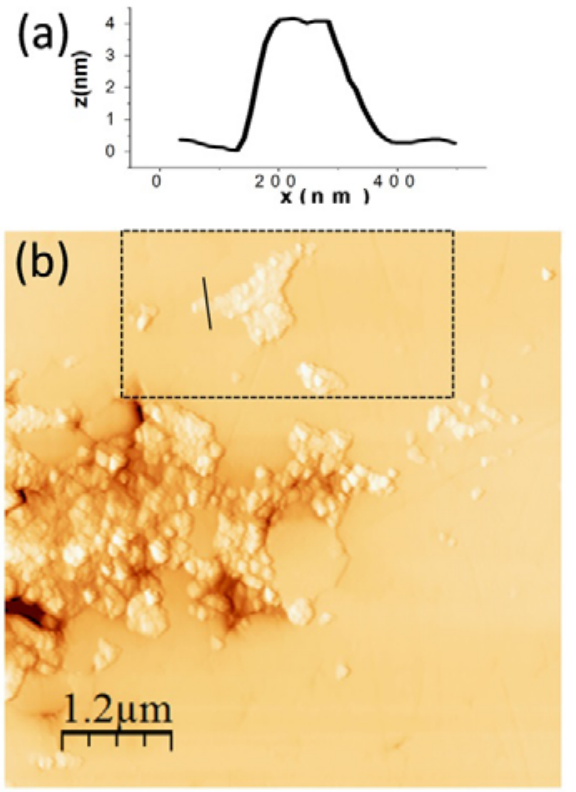

(c)

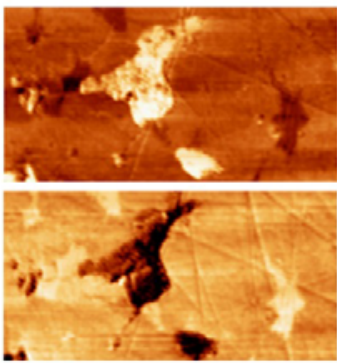

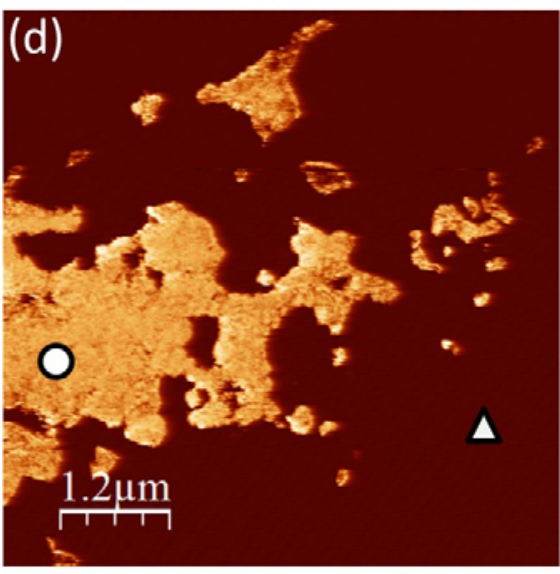

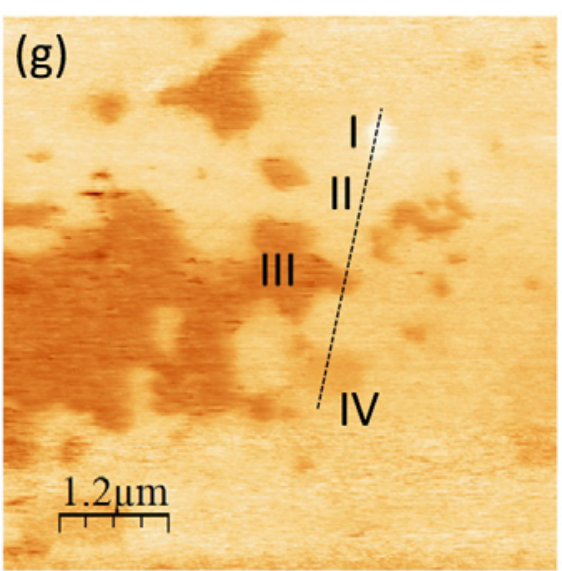

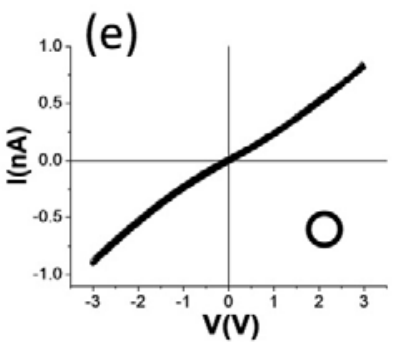

(f)
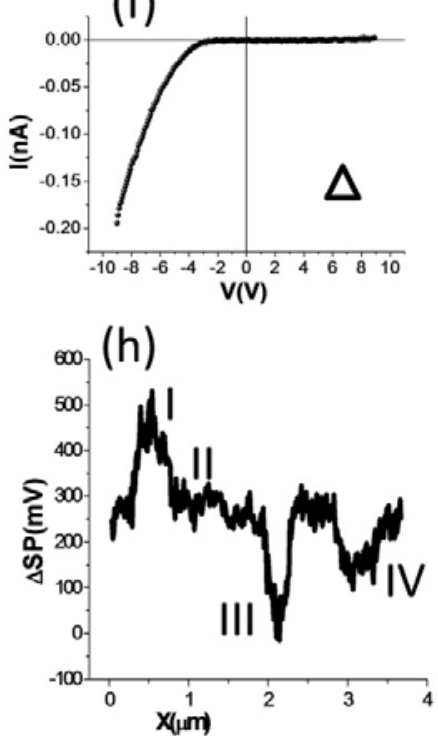

Fig. 3. SFM data series for G/a-SiC composite: (a) topographic relief across a piece of epitaxial graphene flake as indicated by the segment drawn in the topographic image of (b). Total color scale in (b) is $10 \mathrm{~nm}$. (c) Details of the lateral force images (forward and backwards) at the dashed region in (b). (d) Current map at $V_{\text {tip }}=+1 \mathrm{~V}$ with total (black to white) scale $490 \mathrm{pA}$. Images in (b), (c) and (d) were all taken simultaneously in contact mode. (e) and (f) are $\mathrm{I}-\mathrm{V}$ curves obtained after statistically measuring 20 curves at the conducting graphene regions $(0)$ and the primary phase SiC phase $(\Delta)$, respectively. Note the linear and clear n-type rectifying of each case. (g) Surface potential map obtained by KPFM at the same surface area and $(\mathrm{h})$ the corresponding profile crossing the different regions. In KPFM, the $\Delta \mathrm{SP}$ values are measured relative to the B-doped diamond coated probe, i.e. $\Delta \mathrm{SP}=\left(\phi_{\text {tip }}-\phi_{\text {sample }}\right) / \mathrm{e}$.

In Fig. 4 we present the simultaneously topography (Fig. 4a), lateral force (Fig. 4b) (only backwards image) and current map for a bias of $\mathrm{V}_{\text {tip }}=+1.7 \mathrm{~V}$ (Fig. 4c). The current map taken on the same region at the reversed voltage $V_{\text {tip }}=-1.7 \mathrm{~V}$ is also shown (Fig. $4 \mathrm{~d}$ ). At the applied voltages, neither the secondary phase (bright patches in Fig. 4b) nor most of the $\alpha$-SiC exhibit any detectable signal in the CSFM maps. Current is only measured in graphene emerging at the grain boundaries (encircled in white at all images in the figure) 
and in some of the SiC grains (micrometric patches in the current images). Interestingly, for a given grain the intensity depends on the bias sign, being higher for $V_{\text {tip }}<0$ (Fig. 4d) than for $\bigvee_{\text {tip }}>0$ (Fig. 4c) in all cases as corresponds to n-type doped SiC. Moreover, different grains exhibit different current values for the same voltage indicating distinct doping levels and suggesting incorporation of dopants during the sintering process.
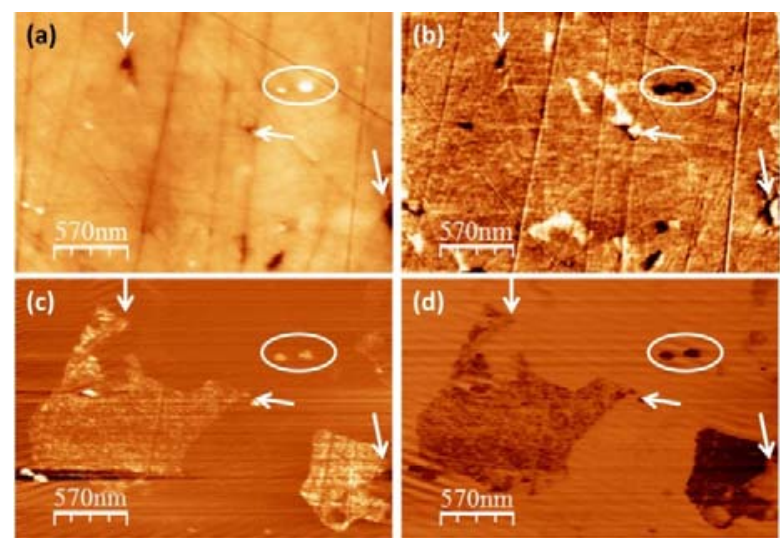

Fig. 4. G/a-SiC composite. Top: Topography (a) and lateral force backwards (b), where brighter means lower friction (see FFM in Supplementary Data). Total topographic scale is $5 \mathrm{~nm}$ (black to white). Bottom: CSFM images acquired at $V_{\text {tip }}=+1.7 \mathrm{~V}$ (c) and $V_{\text {tip }}=-1.7 \mathrm{~V}$ (d). Because the current is measured from tip to sample, positive (bright) or negative (dark) current values are measured for $V_{\text {tip }}>0$ and $V_{\text {tip }}<0$, respectively. Total current scales are $100 \mathrm{pA}$. White circles and arrows are used in all images to indicate graphene flakes and inter-grain pits, respectively.

The possible influence of the grain growth and SiC polytype on the composite nanoscale properties has been addressed by similar measurements on samples obtained from $\beta$-SiC powders. Akin to the $\mathrm{G} / \alpha-\mathrm{SiC}$ case, the graphitic component of the G/ $\beta$-SiC was detected at the $\mathrm{SiC}$ grain boundaries (Fig. 5). The graphene sheets were identified by a large intensity in the current maps at $V_{\text {tip }}=+1 \mathrm{~V}$ (Figs. $5 \mathrm{~d}$ and $5 \mathrm{e}$ ) and a linear I-V response (Fig. 5b). Because the large difference in conductivity between graphene (few $\mu \mathrm{A}$ at + $1 \mathrm{~V}$ ) and the $\mathrm{SiC}$ matrix, no current was detected for the SiC grains (Fig. $5 \mathrm{~d}$ ) unless a high amplifier gain is used (Fig. 5 e). Even at this magnification only $\approx 50-60 \%$ of the SiC grains conduct at the applied voltage ( $\leq$ $400 \mathrm{pA}$ at $+1 \mathrm{~V}$ ). This result points again to the presence of grains with different levels of doping. Compared 
to those for the G/ $\alpha$-SiC (Fig. 3f), I-V curves taken at the $\beta$-SiC grains (Fig. $5 \mathrm{c}$ ) show much lower rectification and slightly more current for positive than for negative voltages ( $p$-type). The observed $p$-doping probably occurs by incorporation of aluminum, coming from either the starting powders $(0.028$ at. $\%$, see Table S1 in the Supplementary Data) or the sintering additives. Despite there is not a significant increase in the mean grain size (i.e. $d_{50}=0.54 \mu \mathrm{m}$ vs. $0.50 \mu \mathrm{m}$ for the starting powder), there is a $25 \%$ increase in the number of grains above $1 \mu \mathrm{m}$ in this $\mathrm{G} / \mathrm{\beta}$-SiC composite [45].
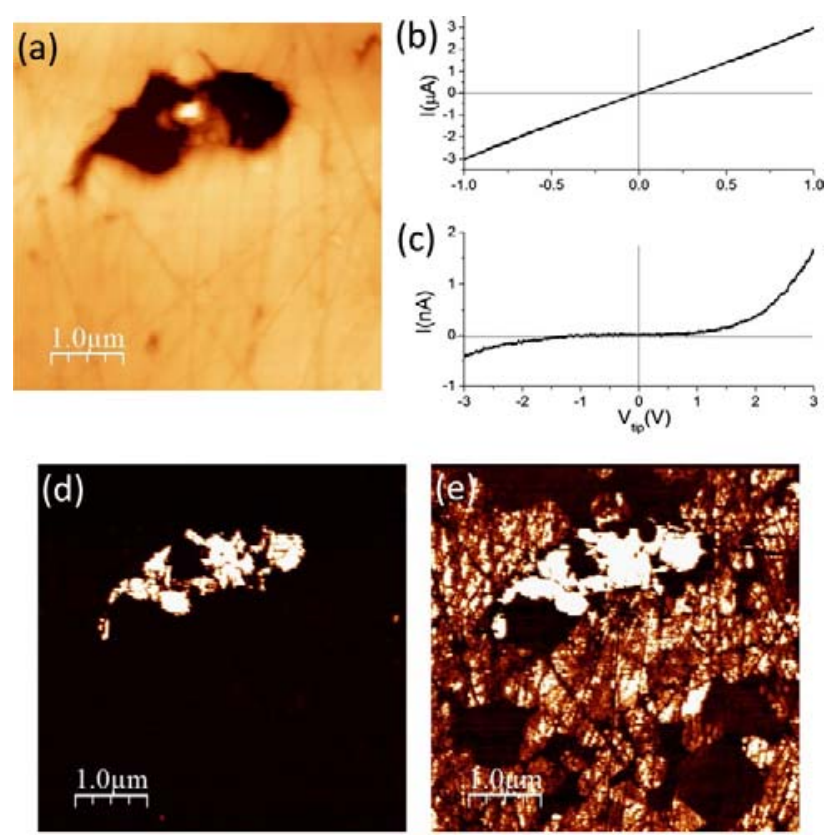

Fig. 5. G/ $\beta$-SiC composite. Top: topography (a) and I-V curves obtained for a voltage range of $\pm 1 \mathrm{~V}$ at a FLG located at the grain boundary (b) and for $\pm 3 \mathrm{~V}$ at the $\beta$-SiC grain surface (c). Bottom: current maps obtained at $V=+1 \mathrm{~V}$ and two different current converter gains, $I_{\max }=10 \mu \mathrm{A}$ (c) and $100 \mathrm{nA}$ (d). Note that high sensitive current measurements evidence the existence of zones with different conductivity (different doping level) in the matrix region. Data were obtained with an Asylum equipment using a Dual Gain ORCA cantilever holder with two IV gains.

Analogous analysis is presented in Fig. 6 for the $\mathrm{G} / \mathrm{n} \beta$-SiC composite, where the topography and simultaneous current data are shown along with KPFM results. Even though presenting different resistivity most $\mathrm{SiC}$ grains do conduct at the applied voltage $(-2 \mathrm{~V})$ except a small percentage of them (see red arrows in the images). These resistive grains (see map of resistance in Fig. S8 of the Supplementary Data) probably correspond to original $\beta$-SiC particles considering their small size; whereas larger ones have 
grown during sintering by a solution-precipitation process. Liquid phase sintered SiC, with some amounts of $\mathrm{Al}_{2} \mathrm{O}_{3}-\mathrm{Y}_{2} \mathrm{O}_{3}$ as sintering aids, leads to materials containing oxygen and $\mathrm{Al} / \mathrm{Y}$ atoms in solid solution within the SiC grains showing the typical core-rim structure [46]. Moreover, some studies [47] have revealed that p-type impurities (e.g. B and $\mathrm{Al}$ ) tend to stabilize the cubic structure $(\beta-S i C)$ which also agrees with present observations. The comparatively higher resistance of the smaller grains points to a semiconductor gap larger than $\pm 2 \mathrm{~V}$ in the starting SiC particles, while grain growth by solution-precipitation seems to reduce this gap. Fig. $6 \mathrm{~d}$ is a typical I-V with a marked p-type rectifying character measured on a SiC grain (see Fig. S9 in the Supplementary Data for I-V curves on different grains). As in the other composites, graphene is perfectly identified at the inter-grain locations (indicated by black arrows in the images) by a high conductivity with currents of few microamperes. However, though quite symmetric in general, and contrary to that observed in the other two composites, the local I-V curves measured at the graphitic regions (Fig. 6c) are clearly non-ohmic, indicating that diverse weighing conduction mechanisms are involved in this case.
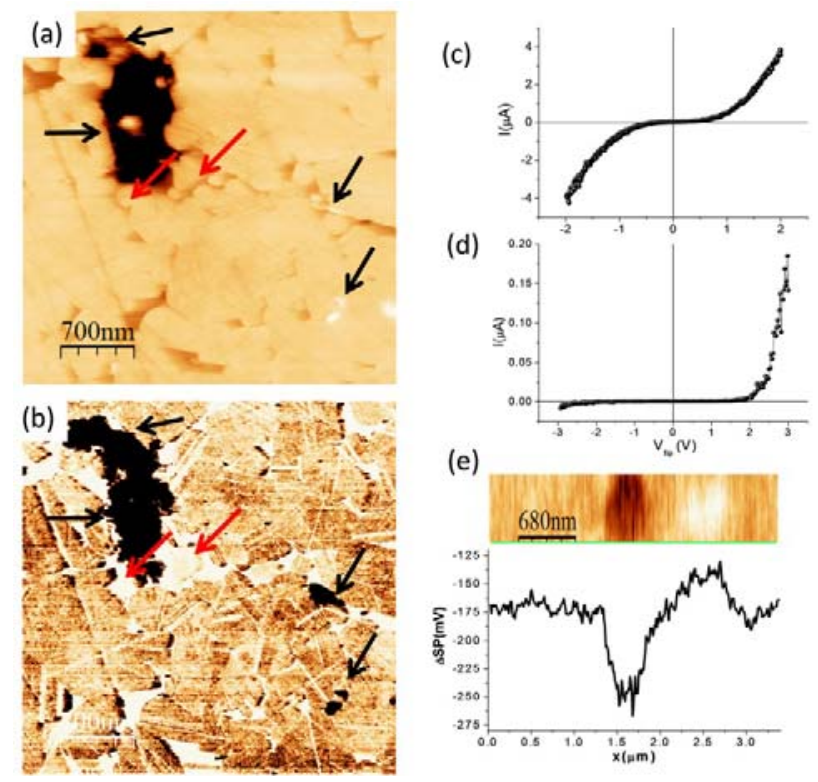

Fig. 6. Graphene/n $\beta$-SiC composite. Left: Topography (a) and current map (b) obtained at $V_{\text {tip }}=-2 \mathrm{~V}$. The total scale of the current map goes from 0 (white) to $-6 \mu \mathrm{A}$ (black). Note in (b) that graphene at the inter-grain regions present the higher conductivity (black arrows), whereas different $\mathrm{SiC}$ grains present slightly different conductivity and some are clearly non-conducting at the applied voltage (see red arrows). Right: I-V curves obtained on top of a graphene flake (c) and a SiC grain (d). (e) Surface potential map obtained by KPFM at a surface region containing the three composite constituents: graphene (dark color), secondary phase (bright color) and $\mathrm{SiC}$ majority phase (intermediate color). In KPFM, the $\Delta S P$ values are relative to the probe, i.e. $\Delta S P=\left(\phi_{\text {tip }}-\phi_{\text {sample }}\right) / e$. 
These results demonstrate that, in this particular $\mathrm{G} / \mathrm{n} \beta-\mathrm{SiC}$ composite, there are two competing conduction paths associated to the graphene and matrix networks, respectively, being the graphene one less resistive. As a consequence, conduction through these connected networks leads to the non-ohmic response measured at graphene flakes (Fig. 6c) conversely to the linear I-V response they show in G/ $\alpha-S i C$ (Fig. 3e) and $\mathrm{G} / \beta$-SiC (Fig. $5 b$ ). A quantitative comparison between the current measured at $+1 \mathrm{~V}$ for each composite on the graphene flakes (IG) and on top of the SiC grains (ISiC) is given in Table 1, both increasing with the electrical conductivity measured at a macroscopic level, $\sigma_{\mathrm{e}}$. Furthermore, IG is found to be $10^{4}$ times higher for $\mathrm{G} / \alpha-\mathrm{SiC}$ than for $\mathrm{G} / \beta-\mathrm{SiC}$ similarly to the corresponding macroscopic $\sigma_{\mathrm{e}}$. However, IG for $\mathrm{G} / \mathrm{n} \beta-\mathrm{SiC}$ is very similar to that measured for $\mathrm{G} / \beta-\mathrm{SiC}$ but its $\sigma_{\mathrm{e}}$ is twenty times higher and, therefore, the conducting $\mathrm{SiC}$ grains must contribute at a macroscopic level in these composites.

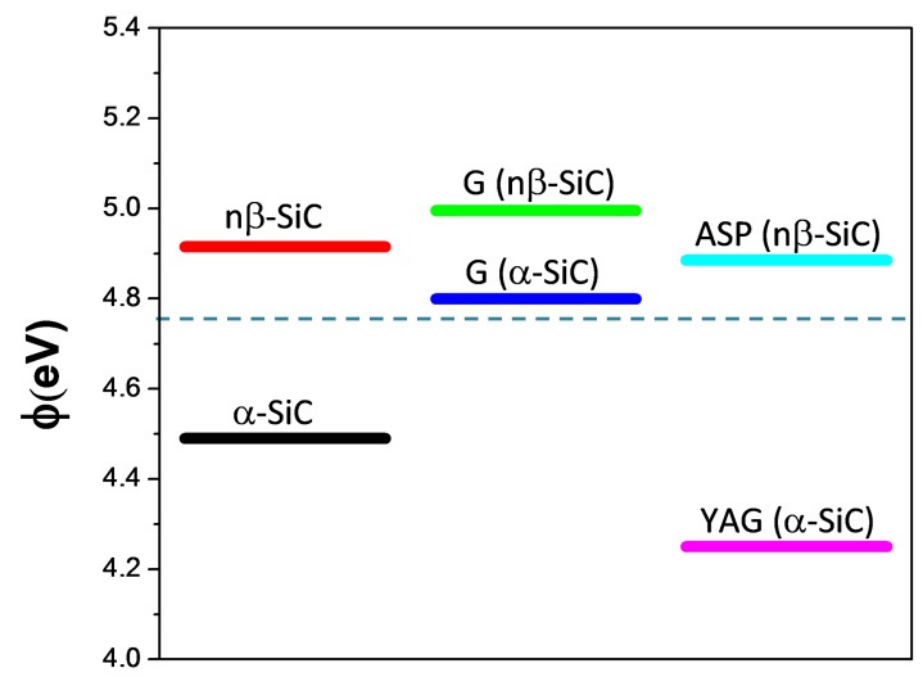

Fig. 7. Work function $(\phi)$ values obtained from KPFM data for each constituent of the $G / \alpha-S i C$ and $G / n \beta-S i C$ composites (ASP stands for amorphous secondary phase). The dashed line corresponds to the work function of the employed B-doped diamond coated tip $(\phi \approx 4.75 \mathrm{eV})$. As commented on the text the values for $\mathrm{SiC}$ depends on the doping level, therefore only the common value of the majority phase is represented.

Fig. 7 summarizes the KPFM data for G/n $\beta$-SiC (Fig. 6e) and G/ $\alpha$-SiC composites (Fig. 3h). The non-ohmic I-V response measured at the graphene locations of $\mathrm{G} / \mathrm{n} \beta-\mathrm{SiC}$ is manifested by a large work function $(\phi \approx$ $5.0 \mathrm{eV}$ ) that contrasts with the obtained for graphene in the $\mathrm{G} / \alpha-\mathrm{SiC}$, with a value of $\phi \approx 4.8 \mathrm{eV}$ close to that 
of the B-doped diamond coated tip. Moreover, all constituents in the $\mathrm{G} / \mathrm{n} \beta$-SiC compound present similar work function, supporting electrical interconnection. The $\phi$ values for the crystalline secondary and majority phases of the G/ $\alpha$-SiC clearly deviate from the graphene counterpart, which is responsible of most conduction in this composite (Table 1). Apart from the opposite rectifying sign of SiC grains in each composite, we remark that the difference in the onset voltage value between both materials is related to their different band gap and, therefore, dependent of the doping level. In consistency with the expected, our results indicate that $\mathrm{n} \beta-\mathrm{SiC}$ has a smaller band gap and higher electron mobility than $\alpha-\mathrm{SiC}$. The above results might be understood either by original doping of the powders or more probably by dopant incorporation during sample fabrication. The fundamental aspects of doping, i.e., the solubility of the impurity atoms, the mechanisms of impurity atom diffusion and incorporation to the lattice are highly dependent of material preparation. On the one hand, all SiC polytypes are susceptible of both $\mathrm{n}$ - and $\mathrm{p}$ doping, being $\mathrm{N}$ and $\mathrm{Al}$ the most common dopants in each case [48]. On the other hand, the carbon enriched silicon carbide $(\beta-\mathrm{SiC})$ is known to be more suitable for $p$-type doping by incorporation of small amount of $\mathrm{Al}$ atoms substituting $\mathrm{Si}$ on the $\mathrm{SiC}$ lattice. The more significant doping observed for the $\mathrm{G} / \mathrm{n} \beta$-SiC composite is likely related to the formation of an Al solid solution during sintering by a solution-precipitation mechanism, as it can be inferred from its higher grain growth. 


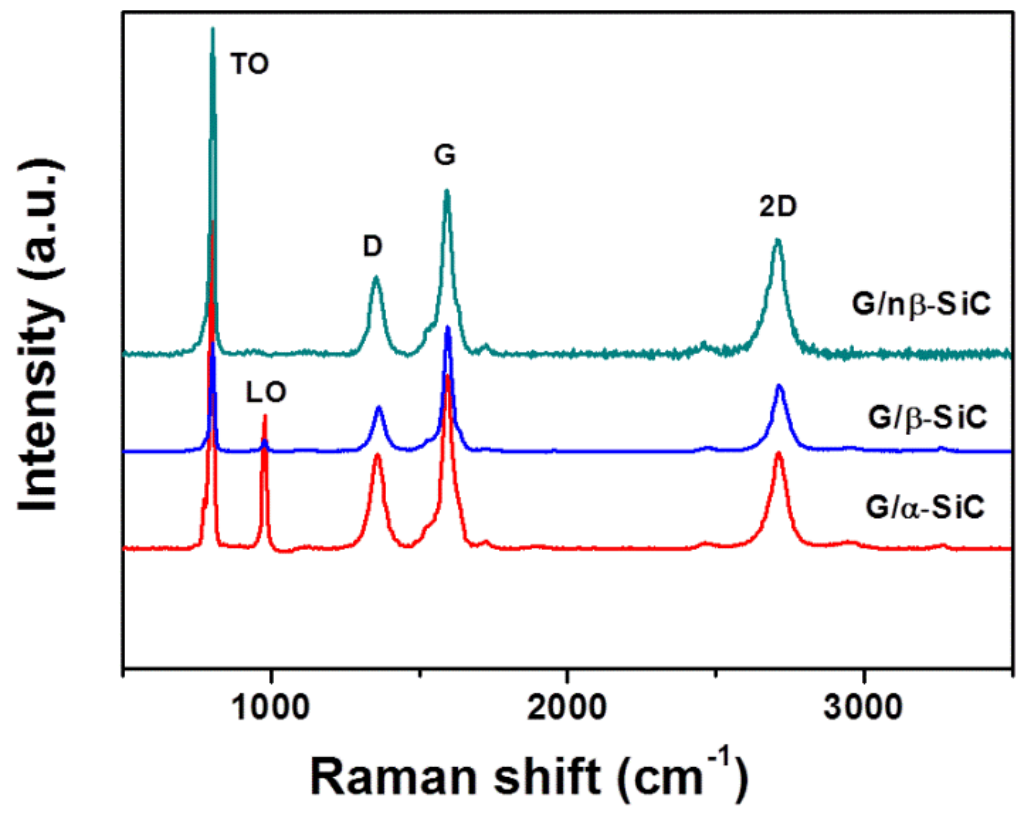

Fig. 8. Micro-Raman average spectra acquired over areas of $10 \mu \mathrm{m} \times 10 \mu \mathrm{m}$ on polished surfaces of the G/ $\alpha$-SiC, $\mathrm{G} / \beta-\mathrm{SiC}$ and $\mathrm{G} / \mathrm{n} \beta-\mathrm{SiC}$ specimens. $60 \times 60$ pixels, one spectrum/pixel were recorded using $100 \mathrm{~ms}$ of acquisition time per spectrum and the $532 \mathrm{~nm}$ laser wavelength excitation.

The Raman analysis presented in Fig. 8 confirms the different level of doping detected by KPFM. The micro-Raman average spectra of the three composite materials show the D-, G- and 2D-bands associated to graphene and two intense peaks centered at $~ 795$ and $969 \mathrm{~cm}^{-1}$, corresponding to the transverse-optical (TO) and longitudinal-optical (LO) modes, respectively, associated to SiC. Collective excitation of free carriers (plasmon) interacts with LO phonon via their macroscopic electric fields to form the LO phonon \pm plasmon coupled mode. In this way, the $964.5 \mathrm{~cm}^{-1}$ and $969.8 \mathrm{~cm}^{-1}$ bands of undoped a-SiC corresponding to the axial and planar coupled modes, respectively, become broadened, less intense and slightly shifted to higher-frequencies [49], as observed in the Table 1, where the intensity ratio between the LO and TO bands (ILO/ITO) clearly decreases for the $\beta$-SiC, especially for the highly doped $\mathrm{G} / \mathrm{n} \beta-\mathrm{SiC}$ where it disappears. 
Table 1. Current measured by CSFM at $\mathrm{V}_{\text {tip }}=+1 \mathrm{~V}$ for each composite on the graphene flakes (IG) and on top of the $\mathrm{SiC}$ grains $(\mathrm{ISiC})$, electrical conductivity measured at a macroscopic level $\left(\sigma_{\mathrm{e}}\right)$ and TO- and LOband positions $(\mathrm{P})$ and ILO/ITO ratio for averaged Raman spectra of the different materials.

\begin{tabular}{|c|c|c|c|c|c|c|}
\hline Material & IG $(A)$ & $\operatorname{ISiC}(\mathrm{A})$ & $\sigma_{e}\left(S \cdot m^{-1}\right)$ & $\operatorname{PLO}\left(\mathrm{cm}^{-1}\right)$ & PTO $\left(\mathrm{cm}^{-1}\right)$ & ILO/ITO \\
\hline $\mathrm{G} / \mathrm{n} \beta-\mathrm{SiC}$ & $1-5 \times 10^{-6}$ & $3 \times 10^{-9}$ & $1.0 \times 10^{2}$ & 977 & 796 & $\mathrm{G} / \mathrm{n} \beta-\mathrm{SiC}$ \\
\hline $\mathrm{G} / \beta-\mathrm{SiC}$ & $3 \times 10^{-6}$ & $4 \times 10^{-10}$ & $5.5 \times 10^{0}$ & 981 & 801 & G/B-SiC \\
\hline G/a-SiC & $3 \times 10^{-10}$ & $<10^{-11}$ & $6.4 \times 10^{-4}$ & ------- & 804 & G/a-SiC \\
\hline
\end{tabular}

\section{Conclusions}

The extensive nanoscale investigation presented here for a series of new graphene/ceramic composites reveals stunning local properties not evident from the composites macroscopic characteristics, which nevertheless depend on them. The combination of different local probe microscopies results a powerful tool, which allows both compound and site recognition of the different phases coexisting on the composite and permits unambiguously determining functional properties at the local scale. The arrangement of the graphitic layers inside the composite surrounding the ceramic matrix grains and forming a conducting three dimensional network or percolated circuits is evidenced.

A noticeable difference in n-type or $p$-type character of the $\mathrm{SiC}$ matrix in the investigated $\alpha$-SiC or $\beta$-SiC and $\mathrm{n} \beta$-SiC composites is interpreted in terms of the different growth kinetics during sintering. A larger grain kinetics growth is accompanied by an increase in the solution-precipitation process promoting $p$-doping. The type and level of doping change the local properties of $\mathrm{SiC}$ and, as a consequence, those of the composite through the ohmic interconnection of the graphene component. By analyzing differences in local current 
data for the composite components a clear correlation with the macroscopic conductivity is established, being demonstrated that current flows mainly through the graphene network for the low doped materials. High doping during sintering occurs in the case of the nano $\beta$-SiC and therefore, a current path through the $\mathrm{SiC}$ matrix competes with the graphene network.

Results obtained for composites derived from different SiC polytypes emphasize the relevance for materials engineering of using novel sintering procedures, which eventually permit tailoring the doping type and carrier concentration of the wide band-gap semiconductor SiC, issues of crucial importance for high power and high temperature applications. Moreover, the strong experimental indication of the role of the graphene constituent in the formation of ohmic contacts in $\mathrm{SiC}$, prerequisite for diodes manufacturing, makes these composite materials suitable candidates for electronic devices at the nanoscale.

\section{Acknowledgements}

This work has been supported by the Spanish Government under projects MAT2013-47869-C4-1-P, MAT2012-32944 and CSIC (PIE201360E063). C. O acknowledges the Spanish MINECO through the Severo Ochoa Program (SEV-2015-0496) and the Generalitat de Catalunya through 2014 SGR 501. L. LM and B. RM thank the Spanish MINECO for FPI BES-2013-063424 and FPI BES-2010-041382 FPI fellowships. The authors acknowledge L. Garzón for his help with some SFM data.

\section{Appendix A. Supplementary data}




\section{References}

[1] K. A Schwetz, In: Handbook of ceramic hard materials. Riedel R, Ed. Weinheim: Wiley-VCH Verlag GmbH, Weinheim, 2000, pp 683-748.

[2] G. Roewer, U. Herzog, K. Trommer, E. Müller, S. Frühauf, Silicon carbide-a survey of synthetic approaches, properties and applications, Struct. Bond. 101 (2002) 59-135.

[3] S. Chattopadhyay, A.L. Lipson, H.J. Karmel, J.D. Emery, T.T. Fister, P.A. Fenter, M.C. Hersam, M.J. Bedzyk, In Situ X-ray Study of the Solid Electrolyte Interphase [SEI] Formation on Graphene as a Model Liion Battery Anode, Chem. Mater. 24 (2012) 3038-304.

[4] H. Li, H. Yu, X. Zhang, G. Guo, J. Hu, A. Dong, D. Yang, Bowl-Like 3C-SiC Nanoshells Encapsulated in Hollow Graphitic Carbon Spheres for High-Rate Lithium-lon Batteries, Chem. Mater. (2016) DOI: 10.1021/acs.chemmater.5b04750

[5] Y. Zhu, S. Murali, W. Cai, X. Li, J.W. Suk, J.R. Potts, R.S. Ruoff, Graphene and Graphene Oxide: Synthesis, Properties, and Applications, Adv. Mater. 22 (2010) 3906-3924.

[6] L. Zhang, Z. Shi, Y. Wang, R. Yang, D. Shi, G. Zhang, Catalyst-free growth of nanographene films on various substrates, Nano Res. 4(2011) 315-321.

[7] D. Pierucci, H. Sediri, M. Hajlaoui, E. Velez-Fort, Y.J. Dappe, M.G. Silly, R. Belkhou, A. Shukla, F. Sirotti, N. Gogneau, A. Ouerghi, Self-organized metal-semiconductor epitaxial graphene layer on off-axis 4H-SiC[0001], Nano Res. 8 (2015) 1026-1037.

[8] P. Miranzo, C. Ramírez, B. Román-Manso, L. Garzón, H.R. Gutiérrez, M. Terrones, C. Ocal, M.I. Osendi, M. Belmonte, In situ processing of electrically conducting graphene/SiC nanocomposites, J. Eur. Ceram. Soc. 33 (2013) 1665-1674.

[9] B. Román-Manso, E. Sánchez-González, A.L. Ortiz, M. Belmonte, M.I. Osendi, P. Miranzo, Contactmechanical properties at pre-creep temperatures of fine-grained graphene/SiC composites prepared in situ by Spark Plasma Sintering, J. Eur. Ceram. Soc. 34 (2014) 1433-1438. 
[10] L. Aballe, S. Matencio, M. Foerster, E. Barrena, F. Sánchez, J. Fontcuberta, C. Ocal, Instability and Surface Potential Modulation of Self-Patterned [001] $\mathrm{SrTiO}_{3}$ Surfaces, Chem. Mat. 27 (2015) 6198-6204.

[11] C. Ocal, R. Bachelet, L. Garzón, M. Stengel, F. Sánchez, J. Fontcuberta, Nanoscale laterallymodulated properties of oxide ultrathin films by substrate termination replica through layer-by-layer growth, Chem. Mat. 24 (2012) 4177-4184.

[12] I. Horcas, R. Fernández, J.M. Gómez-Rodríguez, J. Colchero, J. Gómez-Herrero, A.M. Baro, WSXM: A software for scanning probe microscopy and a tool for nanotechnology, Rev. Sci. Instrum. 78 (2007) 013705-1-8.

[13] R.W. Carpick, M. Salmeron, Scratching the surface: fundamental investigations of tribology with atomic force microscopy, Chem. Rev. 97 (1997) 1163-1194.

[14] F. Houzé, R. Meyer, O. Schneegans, L. Boyer, Imaging the local electrical properties of metal surfaces by atomic force microscopy with conducting probes, Appl. Phys. Lett. 69 (1996) 1975-1977.

[15] M. Nonnenmacher, M.P. O’Boyle, H.K. Wickramasinghe, Kelvin probe force microscopy, Appl. Phys. Lett. 58 (1991) 2921-2923.

[16] C. Barth, A.S. Foster, C.R. Henry, A.L. Shluger, Recent Trends in Surface Characterization and Chemistry with High-Resolution Scanning Force Methods, Adv. Mater. 23 (2011) 477-501.

[17] M. Paradinas, L. Garzón, F. Sánchez, R. Bachelet, D.B. Amabilino, J. Fontcuberta, C. Ocal, Tuning the local frictional and electrostatic responses of nanostructured $\mathrm{SrTiO}_{3}$-surfaces by self-assembled molecular monolayers, Phys. Chem. Chem. Phys. 12 (2010) 4452-4458.

[18] M. Paradinas, C. Munuera, Ch. Silien, M. Buck, C. Ocal, Heterogeneous nanotribological response of polymorphic self-assembled monolayers arising from domain and phase dependent friction, Phys. Chem. Chem. Phys. 15 (2013) 1302-1309.

[19] C. Munuera, E. Barrena, C. Ocal, Scanning force microscopy three-dimensional modes applied to conductivity measurements through linear-chain organic SAMs. Nanotechnology 18 (2007) 125505-125511. 
[20] C. Moreno, C. Munuera, S. Valencia, F. Kronast, X. Obradors, C. Ocal, Reversible resistive switching and multilevel recording in $\mathrm{La}_{0.7} \mathrm{Sr}_{0.3} \mathrm{MnO}_{3}$ thin films for low cost nonvolatile memories, Nano Lett. 10 (2010) 3828-3835.

[21] U. Kolitsch, H.J. Seifert, T. Ludwig, F. Aldinger, Phase equilibria and crystal chemistry in the $\mathrm{Y}_{2} \mathrm{O}_{3}-$ $\mathrm{Al}_{2} \mathrm{O}_{3}-\mathrm{SiO}_{2}$ system, J. Mater. Res. 14 (1999) 447-455.

[22] C. Ramirez, L. Garzon, P. Miranzo, M.I. Osendi, C. Ocal, Electrical conductivity maps in graphene nanoplatelet/silicon nitride composites using conducting scanning force microscopy, Carbon 49 (2011) 3873-3880.

[23] T. Filleter, J.L. McChesney, A. Bostwick, E. Rotenberg, K.V. Emtsev, Th. Seyller, K. Horn, R. Bennewitz, Friction and dissipation in epitaxial graphene films, Phys. Rev. Lett. 102 (2009) 086102-1-4.

[24] T. Filleter, R. Bennewitz, Structural and frictional properties of graphene films on SiC [0001] studied by atomic force microscopy, Phys. Rev. B 81 (2010) 155412-1-7.

[25] L.Y. Lin, D.E. Kim, W.K. Kim, S.C. Jun, Friction and wear characteristics of multi-layer graphene films investigated by atomic force microscopy, Surf. Coat. Technol. 205 (2011) 4864-4869.

[26] H. Lee, N. Lee, Y. Seo, J. Eom, S.W. Lee, Comparison of frictional forces on graphene and graphite. Nanotechnology 20 (2009) 325701-1-6.

[27] C. Lee, Q. Li, W. Kalb, X.Z. Liu, H. Berger, R.W. Carpick, J. Hone, Frictional characteristics of atomically thin sheets. Science 328 (2010) 76-80.

[28] D. Ziegler, P. Gava, J. Güttinger, F. Molitor, L. Wirtz, M. Lazzeri, M.A. Saitta, A. Stemmer, F. Mauri, C. Stampfer, Variations in the work function of doped single-and few-layer graphene assessed by Kelvin probe force microscopy and density functional theory, Phys. Rev. B 83 (2011) 235434-1-7.

[29] S.S. Datta, D.R. Strachan, E.J. Mele, A.T.C. Johnson, Surface potentials and layer charge distributions in few-layer graphene films. Nano Lett. 9 (2009) 7-11. 
[30] R. Wang, S. Wang, D. Zhang, Z. Li, Y. Fang, X. Qiu, Control of carrier type and density in exfoliated graphene by interface engineering, ACS Nano 1 (2011) 408-412.

[31] A. Castellanos-Gomez, R.H.M. Smit, N. Agrait, G. Rubio-Bollinger, Spatially resolved electronic inhomogeneities of graphene due to subsurface charges, Carbon 50 (2012) 932-938.

[32] R. Pearce, J. Eriksson, T. lakimov, L. Hultman, A.L. Spetz, R. Yakimova, On the differing sensitivity to chemical gating of single and double layer epitaxial graphene explored using scanning Kelvin probe microscopy. ACS Nano 7 (2013) 4647-4656.

[33] A. E. Curtin, M.S. Fuhrer, J.L. Tedesco, R. L. Myers-Ward, C.R. Jr. Eddy, D.K. Gaskill, Kelvin probe microscopy and electronic transport in graphene on $\mathrm{SiC}[0001]$ in the minimum conductivity regime, Appl. Phys. Lett 98 (2011) 243111-1-3.

[34] T. Filleter, K.V. Emtsev, Th. Seyller, R. Bennewitz, Local work function measurements of epitaxial graphene, Appl. Phys. Lett. 93 (2008) 133117-1-3.

[35] T. Burnett, R. Yakimova, O. Kazakova, Mapping of local electrical properties in epitaxial graphene using electrostatic force microscopy. Nano Lett. 11 (2011) 2324-2328.

[36] O. Kazakova, T.L. Burnett, J. Patten, L. Yang, R. Yakimova, Epitaxial graphene on SiC[000ㅊ] ]: functional electrical microscopy studies and effect of atmosphere, Nanotechnology 24 (2013) 215702-1-9.

[37] J.M. Mativetsky, E. Treossi, E. Orgiu, M. Melucci, G.P. Veronese, P. Samori, V. Palermo, Local current mapping and patterning of reduced graphene oxide. J. Am. Chem. Soc. 132 (2010) 14130-14136.

[38] J. M. P. Alaboson, Q.H. Wang, J.A. Kellar, J. Park, J.W. Elam, M. Pellin, M.C. Hersam, Conductive atomic force microscope nanopatterning of epitaxial graphene on SiC [0001] in ambient conditions, Adv. Mater. 23 (2011) 2181-2184.

[39] S.H. Ji, J. B. Hannon, R.M. Tromp, V. Perebeinos, J. Tersoff, F.M. Ross, Atomic-scale transport in epitaxial graphene, Nat. Mat. 11 (2012) 114-119. 
[40] P.N. Nirmalraj, T. Lutz, T.; S. Kumar, G.S. Duesberg, J.J. Boland, Nanoscale mapping of electrical resistivity and connectivity in graphene strips and networks, Nano Lett. 11 (2011) 16-22.

[41] M. Lanza, Y. Wang, T. Gao, A. Bayerl, M. Porti, M. Nafria, Y. Zhou, G. Jing, Y. Zhang, Z. Liu, D. Yu, H. Duan, Electrical and mechanical performance of graphene sheets exposed to oxidative environments, Nano Res. 6 (2013) 485-495.

[42] A. Alekseev, D. Chen, E.E. Tkalya, M.G. Ghislandi, Y. Syurik, O. Ageev, J. Loos, G. de With, Local organization of graphene network inside graphene/polymer composites, Adv. Funct. Mater. 22 (2012) 13111318.

[43] J. González-Julián, Y. Iglesias, A.C. Caballero, M. Belmonte, L. Garzón, C. Ocal, P. Miranzo, M.I. Osendi, Multi-scale electrical response of silicon nitride/multi-walled carbon nanotubes composites, Comp. Sci. Technol. 71 (2011) 60-66.

[44] K.S. Krishnan, S.C. Jain, Thermionic constants of graphite. Nature 169 (1952) 702-703.

[45] B. Román-Manso, M. Belmonte, M.I. Osendi, P. Miranzo, Effects of current confinement on the spark plasma sintering of silicon carbide ceramics, J. Am. Ceram. Soc. 98 (2015) 2745-2753.

[46] L.S. Sigl, H.J. Kleebe, Core/Rim structure of liquid-phase-sintered silicon carbide. J. Am. Ceram. Soc. 76 (1993) 773-776.

[47] N.W. Jepps, T.F. Page, In: Progress in Crystal Growth and Characterization; Krishna, P., Eds.; Pergamon: Oxford 7 (1983) pp 259-307.

[48] R. Weingärtner, P.J. Wellmann, M. Bickermann, D. Hofmann, T.L. Straubinger, A. Winnacker, Determination of charge carrier concentration in $\mathrm{n}$ - and $\mathrm{p}$-doped SiC based on optical absorption measurements, Appl. Phys. Lett. 80 (2002) 70-72.

[49] S. Nakashima, H. Harima, Raman investigation of SiC polytypes, Physica Status Solidi [a] 162 (1997) $39-64$. 


\section{Supplementary information}

\section{for Journal of the European Ceramic Society}

Prominent local transport in silicon carbide composites containing in-situ synthesized three-dimensional graphene networks

P. Miranzo, ${ }^{1 *}$ L. López-Mir, ${ }^{2}$ B. Román Manso, ${ }^{1}$ M. Belmonte, ${ }^{1}$ M.I. Osendi ${ }^{l}$ and C. Ocal ${ }^{2 *}$

${ }^{1}$ Instituto de Cerámica y Vidrio (ICV-CSIC) Cantoblanco, 28049-Madrid, Spain

${ }^{2}$ Institut de Ciència de Materials de Barcelona (ICMAB-CSIC)

Campus UAB, 08193 Bellaterra, Barcelona, Spain

*E-mail: pmiranzo@icv.csic.es, cocal@icmab.es 


\section{EXPERIMENTAL DETAILS AND PROCEDURES}

Friction Force Microscopy (FFM). During tip scanning in SFM contact mode operation, in addition to cantilever normal deflection (topography), the cantilever torsion is monitored as a lateral deflection of the reflected laser beam. Since the lateral force caused by friction always opposes the tip motion, the tip twist reverses when the scan direction is inverted. Consequently, if a surface consists of regions with different friction, lateral force images will present opposite contrast in forward and backward directions. Those regions of lower friction would appear as dark patches in the forward and brighter in the backwards scans, respectively. The local friction signal is defined as half the amplitude of the so called friction loop, $\mathrm{F}=1 / 2\left(F \mathrm{l}_{\mathrm{f}}-F \mathrm{l}_{\mathrm{b}}\right)$, where $F \mathrm{l}_{\mathrm{i}}$ is the lateral force signal of the forward $(\mathrm{i}=\mathrm{f})$ and the corresponding backward $(\mathrm{i}=\mathrm{b})$ scans. This line by line procedure can be also applied to the complete lateral force images leading to FFM images or friction maps.

Conducting Scanning Force Microscopy (CSFM). In CSFM, the conducting tip acts as a movable electrode which is placed in direct contact with the sample under controlled load, i.e. by using a normal force feedback, while measuring the current between tip and sample. In our set-up, the current is measured between the biased SFM tip and a metallic counterelectrode clamp attached to the sample and directly contacted to ground. An external I-V converter (Stanford Research Systems) was used to access a wide range of compliance currents $(1 \mathrm{pA}$ to $1 \mathrm{~mA})$. The conducting response of the samples was obtained following two different strategies: i) simultaneously acquiring topographic images $\mathrm{z}(\mathrm{x}, \mathrm{y})$ and current maps I (x,y) over a given region at a given voltage, and ii) acquiring I-V curves at selected $(\mathrm{x}, \mathrm{y})$ locations on the surface (e.g. different composition regions). This procedure allowed localization of the conductive GNP phase and quantification of current transport through it. We note, however, that though in all CSFM experiments the current is measured, it is not itself the magnitude of interest and, moreover, it depends on the contact area, which varies from tip to tip and depends as well on topographic aspects of the surface and the applied force. For this reason, slightly different current values may be obtained for the same voltage as a result of differences in the set points used for the image acquisitions. The way to overcome the current and resistance dependence on contact area is performing comparative analysis from measurements made using the same tip. These comparisons do not provide absolute or intrinsic quantities but serve to elucidate among relative behaviors of different 
surface regions and graphene contents. Moreover, because this approach is only valid if the tip does not change significantly, tip-sample conditions were verified prior to and after each conductivity experiment by a systematic determination of the adhesion force from force versus distance curves. For reliable data comparison, the same tip has been used in all the CSFM experiments of at least one series of samples.

Kelvin probe force microscopy (KPFM). KPFM is a dynamic mode which measures the so called contact potential difference or Surface Potential (SP) through measurement of the electrostatic force between a metallic tip and the sample. Contrary to CSFM, during KPFM the tip and sample are not in direct contact but electrically connected via electronics. As a result, their Fermi levels align creating a SP and thus a tip-sample electrostatic force develops. Though the method has been applied to a wide variety of materials, including insulators, KPFM was first introduced to investigate the work function of metals and it is understood as follows. If the work function of the KPFM tip (vibrating electrode) is $\varphi_{t i p}$ and $\varphi_{\text {sample }}$ is that of the sample, then the contact potential difference between tip and sample is $\mathrm{SP}_{\text {sample }}=\left(\varphi_{\text {tip }}-\varphi_{\text {sample }}\right) / e=\Delta \varphi_{s} / e$, where $e$ is the electric charge. The tip, which is at some distance $(\sim 15 \mathrm{~nm})$ above the sample surface, is driven by an oscillating voltage $\mathrm{V}_{\text {tip }}=\mathrm{V}_{\mathrm{dc}}+$ $\mathrm{V}_{\mathrm{ac}} \sin (\omega \mathrm{t})$ and, as a consequence, the electrostatic force between them is $F_{e s}=$ $-\frac{1}{2} \frac{\partial C}{\partial z}\left[\left(V_{d c}-\Delta \varphi_{s} / e\right)+V_{a c} \sin (\omega t)\right]^{2}$, such that the $\mathrm{V}_{\mathrm{dc}}$ nullifying the first harmonic of this electrostatic force is just the $\mathrm{SP}_{\text {sample }}$. Often, as in the present work, in order to minimize cantilever contributions, the same minimization procedure is used but applied to the force gradient measurement:

$$
\frac{\partial F_{\omega}}{\partial z}=-\frac{\partial^{2} C}{\partial z^{2}}\left[\left(V_{d c}-\Delta \varphi_{s} / e\right)+V_{a c} \sin (\omega t)\right]^{2}
$$

In principle, knowing $\phi_{t i p}, \phi_{\text {sample }}$ can be calculated, but if the sample surface has regions with different electronic properties, as the case presented here, the contact potential of each region would be given by $\mathrm{SP}_{A}=\left(\varphi_{t i p}-\varphi_{A}\right) / e$ and $\mathrm{SP}_{B}=\left(\varphi_{t i p}-\varphi_{B}\right) / e$, respectively. Consequently, the contact potential difference between them is $\Delta \mathrm{SP}=\mathrm{SP}_{A}-\mathrm{SP}_{B}=-\left(\varphi_{A}\right.$ $\left.-\varphi_{B}\right)$ independently of the material the tip is made of. Therefore, the contrast of the surface potential maps obtained by scanning the tip over the surface directly reflects the local variations of the surface work function. Note that by definition, the higher the local SP the 
lower the local effective work function $\left(\Delta \mathrm{SP}=-\Delta \varphi_{A B}\right)$. The method lacks of the desired lateral resolution for measuring nanostructures but avoids problems derived from tip details (end atoms and geometry) appearing when attempting to determine absolute SP values.

Whereas for topographic, lateral force and surface potential images the color code is the commonly used, bright for high and dark for low, for the current maps it depends on the voltage sign. Thus higher currents appear darker in CSFM images taken at negatives $\mathrm{V}_{\text {tip }}$, while brighter for positives $\mathrm{V}_{\text {tip }}$. Note that the joint use of KPFM and CSFM (and FFM) for characterizing the same surface locations guaranties local properties correlation but is challenging since requires passing from non-contact dynamic conditions at which the tip oscillates at some distance away from the surface to direct tip-surface contact at which the electric current (and friction) between tip and surface is measured. 


\section{FIGURES AND TABLES}
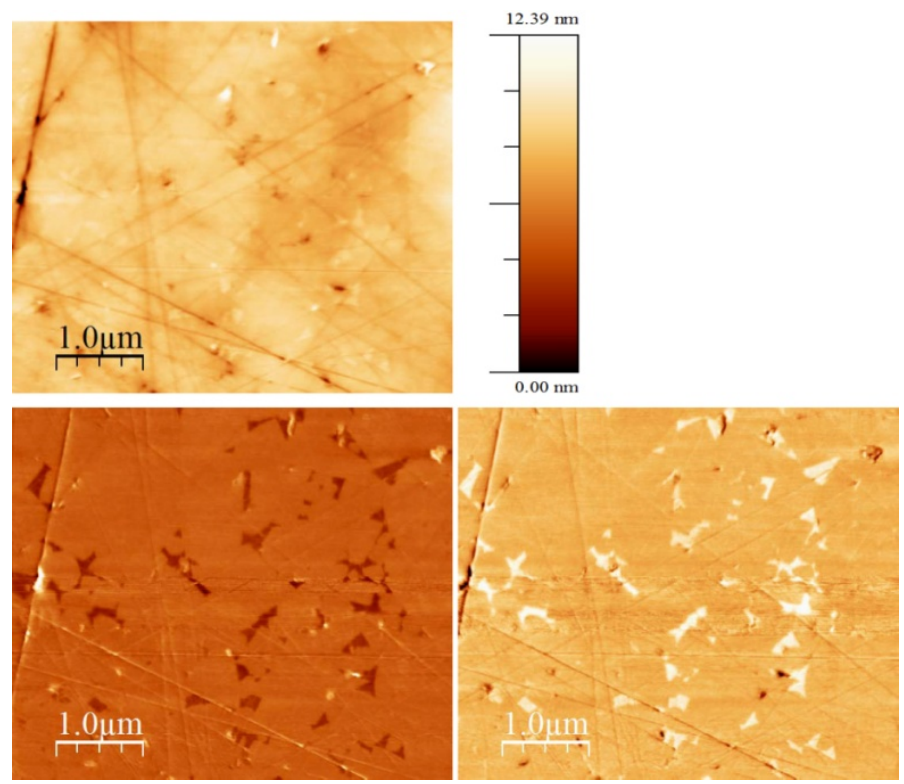

Figure S1. Topography and lateral force images of the graphene/ $\boldsymbol{\alpha - S i C}$ composite. In spite of presenting reminiscences of the polishing (straight lines in the images), the morphology of the as-polished surface is extremely flat (see total scale) with a root means squared roughness of rms $<1 \mathrm{~nm}$ over $25 \mu \mathrm{m}^{2}$. No grain boundaries are detectable in the topographic image but their size and shape could be make out by the shape and distance between secondary phase regions, located at the primary phase grain boundaries as seen by FESEM (see main text). The ratio between secondary (dark/bright in lateral force forward/backward images) and primary phases is about 5\% (average using different images taken over large areas) is also in agreement with these observations. These results reflect the extremely sensitive dependence of FFM on the materials structure and surface composition. This observation is important since it is known that the mechanical properties of composite materials are not merely determined by the intrinsic bulk properties of the individual components, but are known to particularly depend on specific details related to grain boundaries, grain size and defect density.
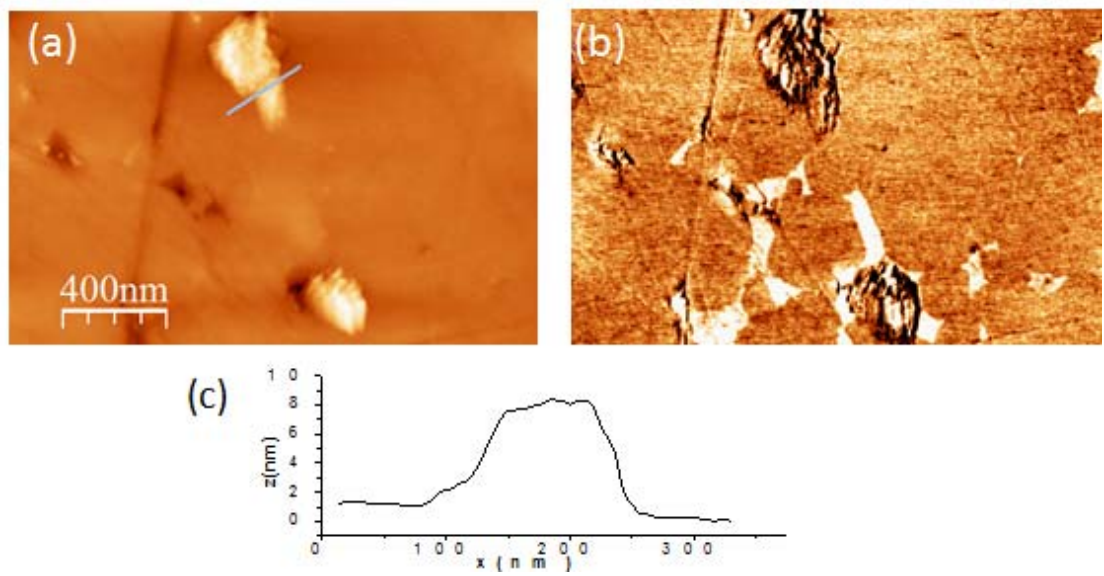

Figure S2. Few layers graphene (FLG). Simultaneously acquired topography (a) and backwards lateral force image (b). (c) Topographic profile across the few layers graphene indicated in (a). See the above FFM experimental procedure description to interpret color code in (b). 

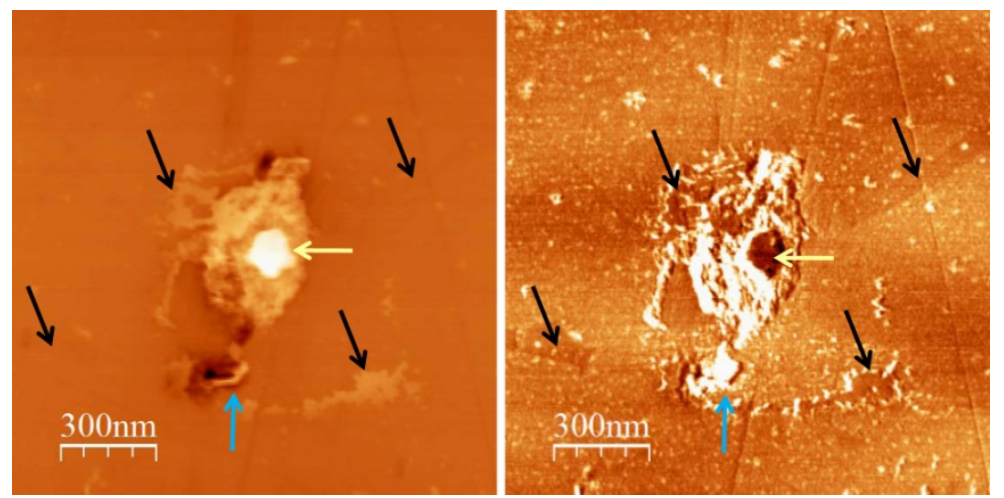

Figure S3. Friction for diverse graphene regions of graphene/ $\alpha-S i C$. Due to the sample preparation for SFM measurements (cutting plus polishing) different types of graphene-based regions exist. Thus, for instance, weak attached FLGs emerging from the grain boundaries (blue arrow) are susceptible of being displaced back and forth during the tip scanning (see figure below). As a consequence of this weak anchoring they exhibit a much higher friction than thin EGL well anchored at the ceramic matrix (black arrows). Moreover, thicker regions show a clearly lower friction (yellow arrows) as expected from graphite.
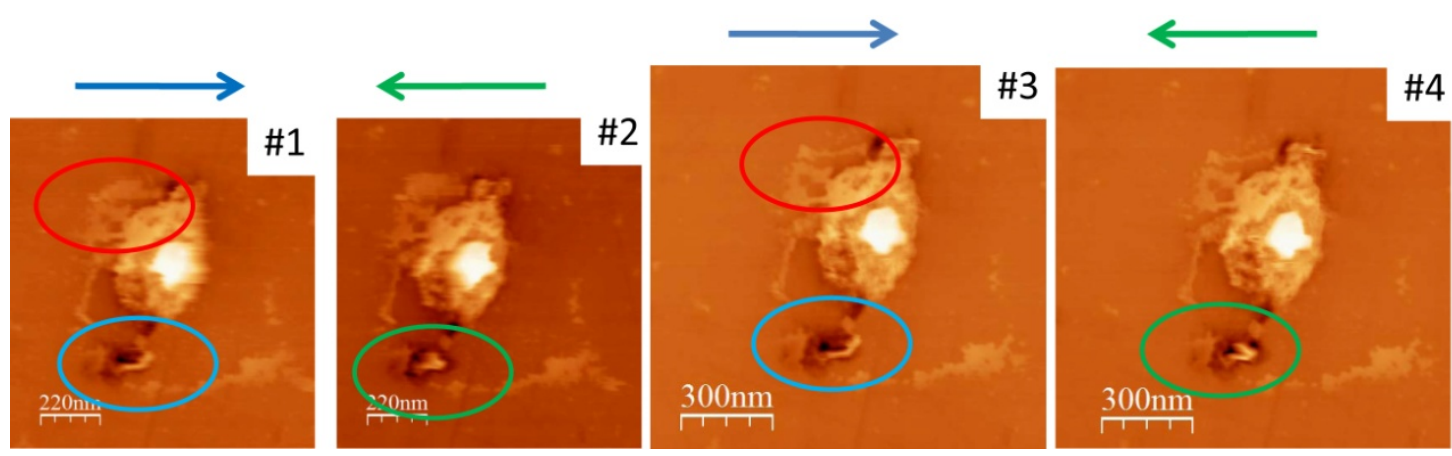

Figure S4. Consecutive topographic images taken in the forward (\#1, \#3) and backward (\#2,\#4) scanning directions. A weakly bound FLG emerging at a grain boundary region of the graphene/ $\alpha$-SiC composite is moved back and forth (circled green and blue, respectively) several times. A thin graphene layer is even removed (circled in red) after some time by the sweeping effect of the tip.
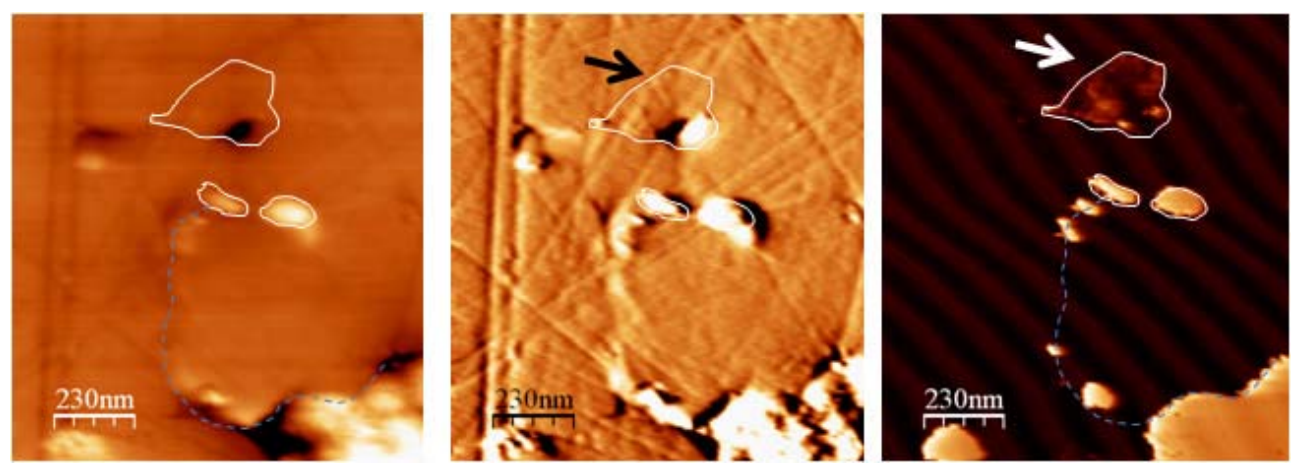

Figure S5. Topography (left), topography derivative (medium) and current map (right) of G/ $\alpha-S i C$. Illustration of diverse FLGs coming up from inter-grain holes and inter-grain boundaries. The FLGs present a high conduction, confirming they form part of the graphene network even if they look disconnected. Note that some areas corresponding to the surface of doped $\mathrm{SiC}$ grains are only observed in the current image. 

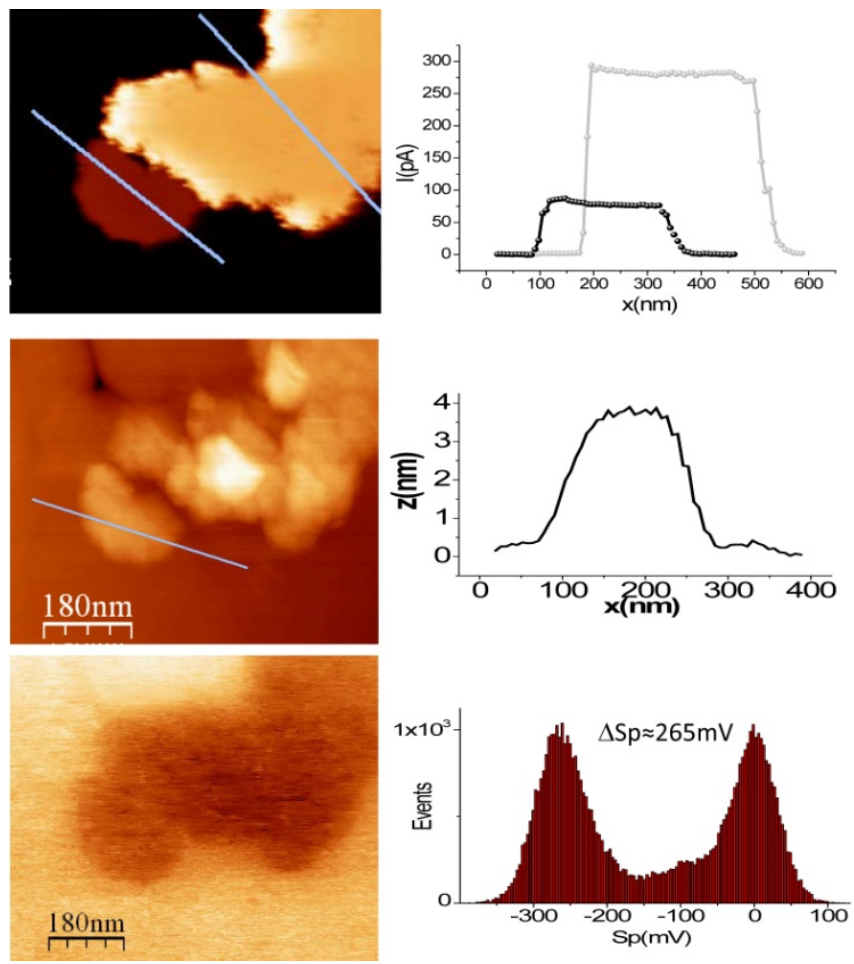

Figure S6. Combined SPM data of graphene/ $\boldsymbol{\alpha}-\mathrm{SiC}$ Top: current map (left) and profiles (right) taken along the segments marked in the current image $\left(V_{\text {tip }}=+1 \mathrm{~V}\right.$, with $\left.\mathrm{I}_{\max }=300 \mathrm{pA}\right)$. Middle: Topography (left) and the corresponding profile (right). Bottom: KPFM image (left) simultaneously measured to the topography shown. A surface potential difference of about $265 \mathrm{mV}$ is measured (histogram at right) between the FLG and the surrounding SiC surface. Note the extremely homogeneous current measured over each FLG. Importantly, this homogeneity stands independently of the local thickness of a given FLG, which indicates an efficient transport between piled layers (low inter-layer resistance). However, the jump in current by a factor of $\sim 3.5$ between adjacent flakes is indicative of the contact resistance between them, i.e. an inter-flake (lateral) resistance which is due to a interrupted continuity of the contact between flakes and visible as a discontinuity in topography.
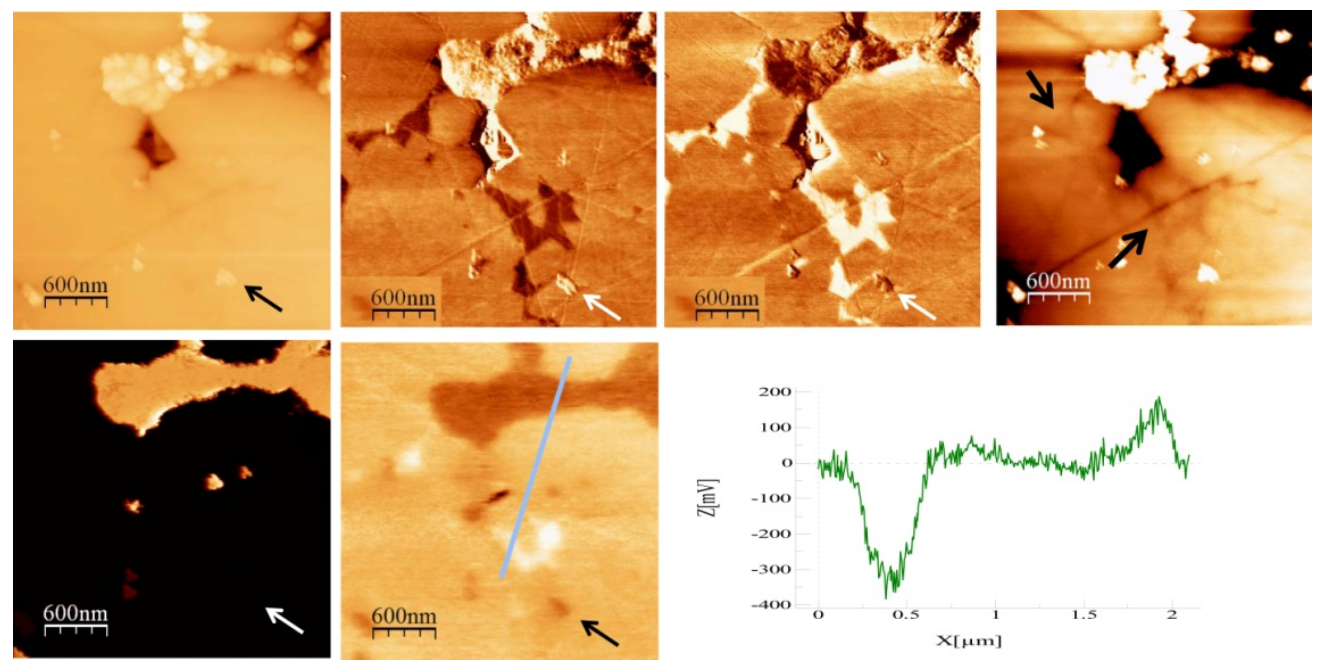

Figure S7. Combined SPM data of graphene/ $\boldsymbol{\alpha}-$ SiC. Top: Topography (a), lateral force forward (b) and backward (c), equalized topography (d). Bottom: CSFM (d) and KPFM (e) images and surface potential profile (f) along the segment in (e). These data were taken on an enlarged area $\left(2.4 \times 2.4 \mu \mathrm{m}^{2}\right)$ of the same surface region shown in Figure 1 of the main manuscript. The equalized topography is presented in (d) to highlight the existence of the secondary phase (black arrows) also visible in the lateral force images and the KPFM maps. 

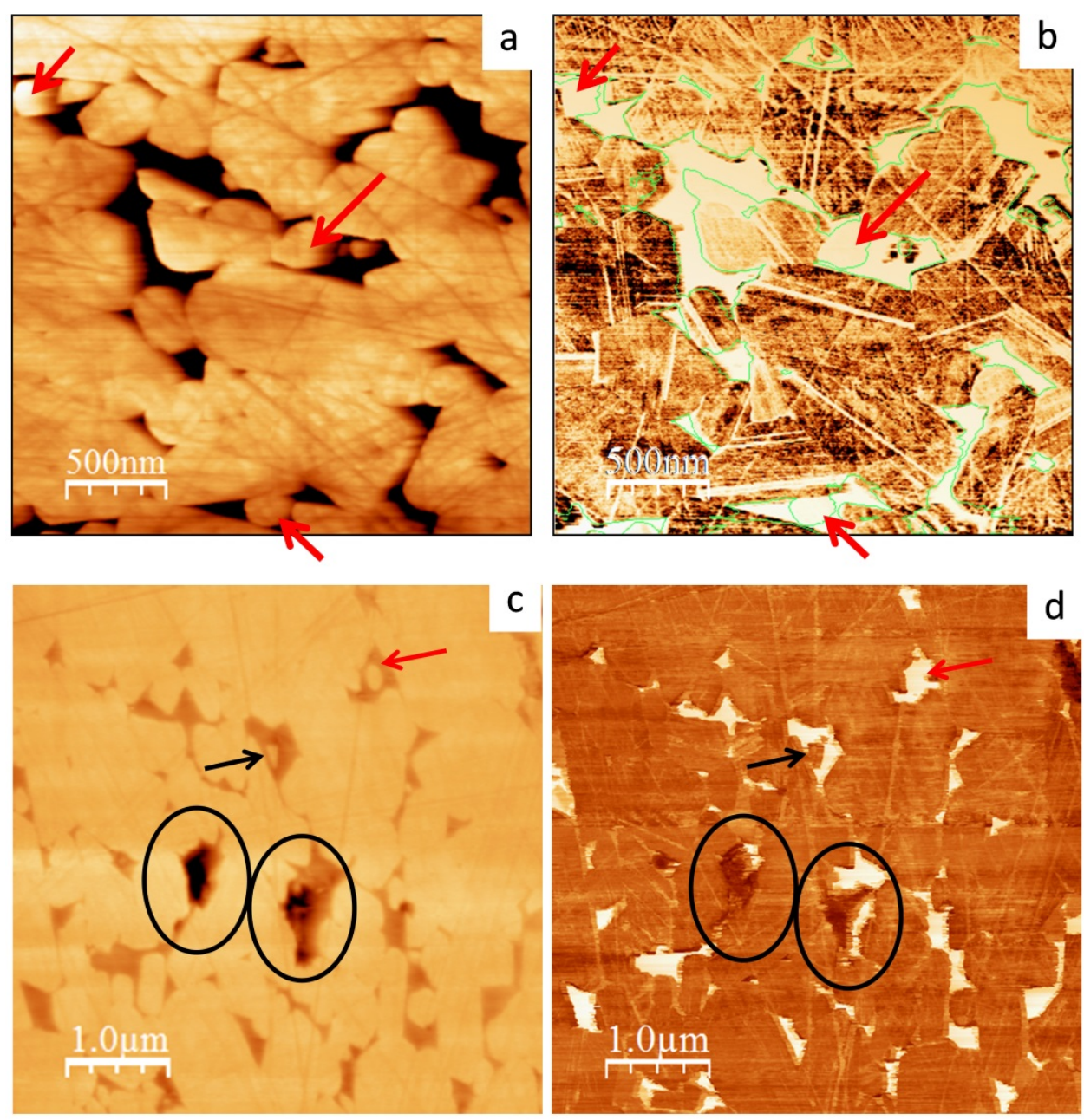

Figure S8. Graphene/n $\boldsymbol{\beta}-\mathrm{SiC}$ composite. Top: (a) Topography and (b) current map obtained at tip bias of $\mathrm{V}_{\text {tip }}=-2 \mathrm{~V}$ at a region with no graphene at the inter-grains. The total scale for the current map ranges from 0 (white) to $-1.5 \mu \mathrm{A}$ (black) and the green countours correspond to the superimposed topographic grain frontiers. Not that different grains present different current value and few are non-conducting (red arrows) at the applied voltage. Bottom: (c) Topography and (d) resistance maps (obtained using a Resiscope Agilent instrument) where the lower the resistance the darker the color. Vertical scales are in arbitrary units in both images that have been equalized. Though the graphene layers are not distinguishable in the topographic image, resistance data reveal their presence at some inter-grain pits circled in (c) and (d). On the other hand, high resistance is measured at some grains (red arrows in the bottom panels). 


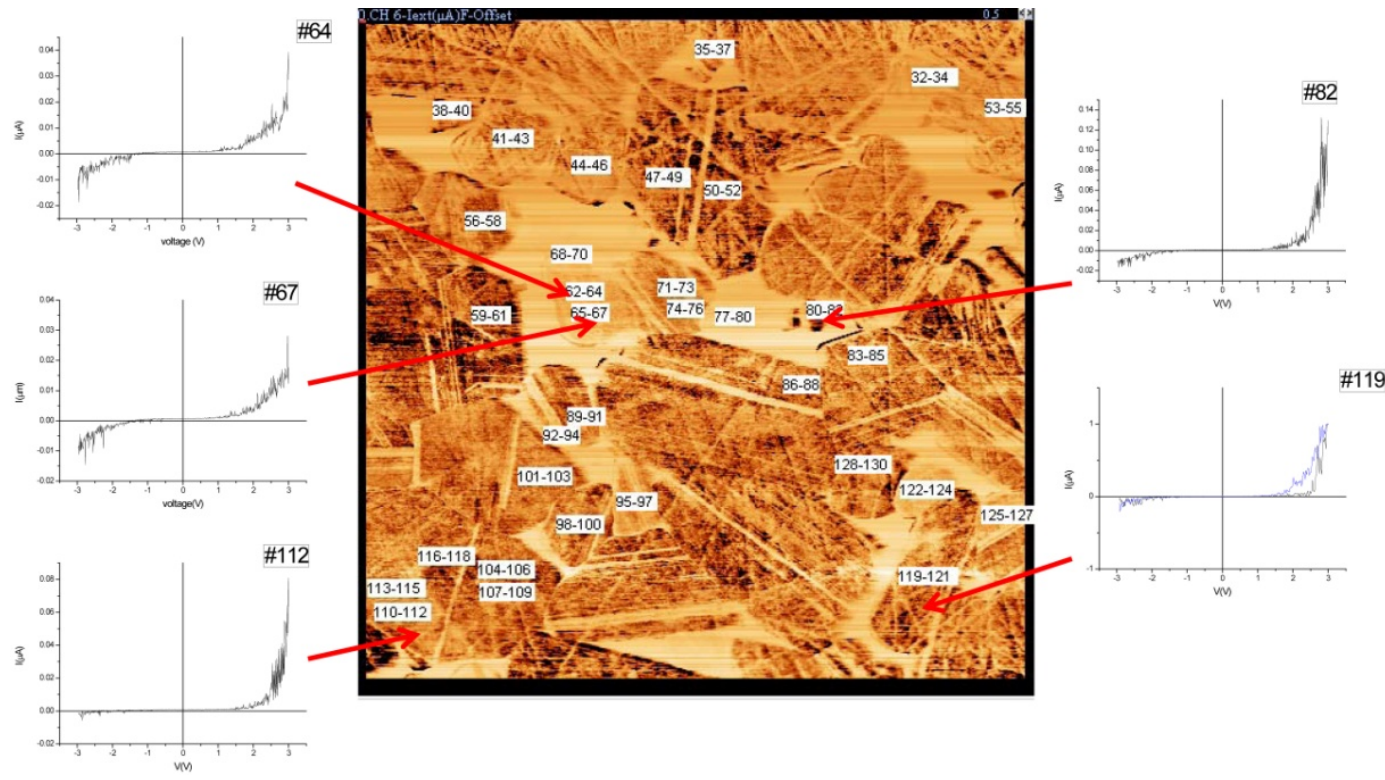

Figure S9. Set of IV curves obtained for the graphene/n $\beta-S i C$ composite. An area avoiding the presence of highly conducting graphene has been chosen for better showing the differences on conductivity of the ceramic matrix. All I-V curves were obtained for $-3.5 \mathrm{~V}<\mathrm{V}<+3.5 \mathrm{~V}$ and their clearly asymmetric shape is ascribed to a p-type doping of $\mathrm{n} \beta$ - $\mathrm{SiC}$ most likely due to $\mathrm{Al}$ incorporation from the initial additives. Depending on the specific grain a different apparent gap is indicative of a different doping level. 
Table S1. Chemical analysis of the different starting SiC powders. All the elements were determined by Xray fluorescence spectroscopy (PANalytical Magix 2400) except the oxygen and nitrogen that were measured by LECO (TC-436)

\begin{tabular}{|c||c|c|c|}
\hline Element & $\begin{array}{c}\alpha-S i C \\
\text { (wt.\%) }\end{array}$ & $\begin{array}{c}\text { B-SiC } \\
\text { (wt.\%) }\end{array}$ & $\begin{array}{c}\text { nano ß-SiC } \\
\text { (wt.\%) }\end{array}$ \\
\hline Al & 0.014 & 0.028 & nd \\
\hline Ti & 0.010 & 0.010 & nd \\
\hline $\mathbf{F e}$ & 0.013 & 0.011 & 0.52 \\
\hline $\mathbf{V}$ & nd & 0.017 & nd \\
\hline $\mathbf{C r}$ & nd & nd & 0.12 \\
\hline $\mathbf{C l}$ & nd & nd & 0.56 \\
\hline $\mathbf{N i}$ & nd & nd & 0.055 \\
\hline $\mathbf{P}$ & nd & nd & nd \\
\hline $\mathbf{N}$ & 0.22 & 0.12 & 0.39 \\
\hline $\mathbf{O}$ & 1.2 & 1.85 & 4.1 \\
\hline
\end{tabular}

